\title{
Latest Inversion-Free Iterative Scheme for Solving a Pair of Nonlinear Matrix Equations
}

\author{
Sourav Shil $(i)$ and Hemant Kumar Nashine \\ Department of Mathematics, School of Advanced Sciences, Vellore Institute of Technology, Vellore-632014, TN, India \\ Correspondence should be addressed to Hemant Kumar Nashine; hemant.nashine@vit.ac.in
}

Received 14 June 2021; Revised 30 July 2021; Accepted 11 August 2021; Published 29 August 2021

Academic Editor: Ali Jaballah

Copyright (c) 2021 Sourav Shil and Hemant Kumar Nashine. This is an open access article distributed under the Creative Commons Attribution License, which permits unrestricted use, distribution, and reproduction in any medium, provided the original work is properly cited.

In this work, the following system of nonlinear matrix equations is considered, $X_{1}+A^{*} X_{1}^{-1} A+B^{*} X_{2}^{-1} B=I$ and $X_{2}+C^{*} X_{2}^{-1} C+$ $D^{*} X_{1}^{-1} D=I$, where $A, B, C$, and $D$ are arbitrary $n \times n$ matrices and $I$ is the identity matrix of order $n$. Some conditions for the existence of a positive-definite solution as well as the convergence analysis of the newly developed algorithm for finding the maximal positive-definite solution and its convergence rate are discussed. Four examples are also provided herein to support our results.

\section{Introduction and Preliminaries}

Consider the system of matrix equations of the form

$$
\begin{array}{r}
\Omega_{1}(X)+A^{*} P_{1}(X) A+B^{*} P_{2}(X) B=Q, \\
\Omega_{2}(X)+C^{*} Q_{1}(X) C+D^{*} Q_{2}(X) D=Q,
\end{array}
$$

where $Q$ is an $n \times n$ Hermitian positive definite matrix (HPD, for short), $A, B, C$, and $D$ are complex matrices of order $n \times n, \Omega_{1}(X), \Omega_{2}(X), P_{1}(X), P_{2}(X), Q_{1}(X)$, and $Q_{2}(X)$ are mappings from the set of positive definite matrices to itself, and $A^{*}$ is the conjugate transpose of $A$.

We can see that the above equations incorporate a few linear as well as nonlinear matrix equations (NMEs, in short). Over the last few decades, many researchers studied systems (1) and (2) with different types of $P_{i}, Q_{i}$, and $\Omega_{i}$, $i=1,2$.

For (1), $\Omega_{1}(X)=X^{s}, P_{1}(X)=X^{-p_{1}}$, and $P_{2}(X)=$ $X^{-} p_{2}, p_{1}, p_{2} \in(0,1]$ and $s \in \mathbb{0}^{+}[1]$

For (1), $\Omega_{1}(X)=X, P_{1}(X)=X^{-1}, P_{2}(X)=X^{-1}$, and $Q=I[2,3]$

For (1), $\Omega_{1}(X)=X, P_{1}(X)=X^{-1}$, and $P_{2}(X)=0[4]$
For (1), $\Omega_{1}(X)=X, P_{1}(X)=X^{-1}$, and $P_{2}(X)=X^{-1}$, $s, t \in \mathbb{N}[5,6]$

For (1), $\Omega_{1}(X)=X^{s}, P_{1}(X)=P^{-p_{1}}$, and $P_{2}(X)=$ $X^{-p_{2}}, s, p_{1}, p_{2} \geq 1[7]$

For (1) and (2), $\Omega_{1}(X)=\Omega_{2}(X)=X, Q_{1}(X)=X^{-} p_{1}$, $P_{1}(X)=X^{-} P_{2}$, and $Q_{2}(X)=Q_{2}(X)=O, Q=I$ and $p_{1}, p_{2} \in \mathbb{Q}^{+}[8]$

For (1) and (2), $\Omega_{1}(X)=\Omega_{2}(X)=X$, $Q_{1}(X)= \pm X^{-} p_{1}, \quad P_{1}(X)= \pm X^{-p_{2}}, \quad$ and $Q_{2}(X)=Q_{2}(X)=O, Q=I$ and $p_{1}, p_{2} \in \mathbb{Q}^{+}[9,10]$

For (1) and (2) $\Omega_{1}(X)=\Omega_{2}(X)=X, P_{1}(X)=X^{-} p_{1}$, $Q_{1}(X)=X^{-p_{2}}$, and $Q_{2}(X)=Q_{2}(X)=O, Q=I$ and $p_{1}, p_{2} \in(0,1][11]$

For different types of applications of the Riccati equation, one can check $[4,12,13]$.

Throughout the article, $\mathbb{R}$ denotes the set of real numbers, $\mathbb{N}$ denotes the set of natural numbers, $\mathbb{R}_{+}=[0,+\infty), \mathbb{a}_{+}$ denotes the set of positive numbers, and $\mathbb{N}^{*}=\mathbb{N} \cup\{0\}$.

$H(n)$ (resp. $K(n)$ and $P(n))$ denotes the set of all $n \times n$ Hermitian (resp. positive semidefinite and positive definite) matrices over $\mathbb{C}$ and $M(n)$ stands for the set of all $n \times n$ matrices over $\mathbb{C}$. 
For a matrix $D \in H(n), s_{1}(D) \geq s_{2}(D) \geq \cdots \geq s_{n}(D)$ denotes its singular values and $|\|D\||$ denotes the sum of these values, i.e., the trace norm of $D$. The Frobenius norm will be denoted by $\|D\|_{F}=\left(\sum_{i, j=1}^{n}\left|D_{i j}\right|^{2}\right)^{1 / 2}$. For $P, Q \in H(n), P \geq Q$ (resp. $P>Q$ ) indicates that $P-Q$ is positive semidefinite (resp. positive definite). $O$ and $I$ stand for the zero and unit matrix in $H(n)$, respectively.

\section{Conditions in Support of the Existence of a Positive Definite Solution}

We begin with two useful lemmas.

Lemma 1 (see [14]). If $P$ and $Q>0$ are Hermitian matrices having the same order, then $2 P-P Q P \leq Q^{-1}$.

Lemma 2 (see [15]). If $U \geq V>O$, then $U^{p} \geq V^{p}>O$, for all $p \in(0,1]$.

Now, let us consider the system of NMEs of the form

$$
\begin{aligned}
X_{1}+A^{*} X_{1}^{-1} A+B^{*} X_{2}^{-1} B & =I, \\
X_{2}+C^{*} X_{2}^{-1} C+D^{*} X_{1}^{-1} D & =I .
\end{aligned}
$$

Taking $Y=X_{1}^{-1}$ and $Z=X_{2}^{-1}$, the system of nonlinear matrix equation (3) is equivalent to

$$
\begin{aligned}
& Y^{-1}+A^{*} Y A+B^{*} Z B=I, \\
& Z^{-1}+C^{*} Z C+D^{*} Y D=I .
\end{aligned}
$$

Theorem 1. Let $(Y, Z)$ be a positive-definite solution (PDS, for short) of (4). Then, $I \leq Y$ and $I \leq Z$.

Proof. Suppose that system (4) has a PDS $(Y, Z)>(O, O)$. Then, from Lemma 2,

$$
\begin{aligned}
& A^{*} Y A+B^{*} Z B \geq O, \\
& C^{*} Z C+D^{*} Y D \geq O,
\end{aligned}
$$

which gives

$$
\begin{aligned}
& Y^{-1}=I-A^{*} Y A-B^{*} Z B \leq I, \\
& Z^{-1}=I-C^{*} Z C-D^{*} Y D \leq I .
\end{aligned}
$$

This, in turn, implies that $I \leq Y$ and $I \leq Z$.

Lemma 3 (see Theorem 2.1 in [16]). Let $U$ and $V$ be positive operators acting on a Hilbert space $H$ in such a way that $P_{1} I \geq U \geq p_{1} I>O, P_{2} I \geq V \geq p_{2} I>O$, and $V \geq U>O$. Then,

$$
\begin{aligned}
& U^{s} \leq\left(\frac{P_{1}}{p_{1}}\right)^{s-1} V^{s}, \\
& U^{s} \leq\left(\frac{P_{2}}{p_{2}}\right)^{s-1} V^{s},
\end{aligned}
$$

hold for any $s \geq 1$.
Remark 1. In the case that $V>U>O$ in Lemma 3, we have $U^{s}<\left(P_{1} / p_{1}\right)^{s-1} V^{t}$ and $U^{s}<\left(P_{2} / p_{2}\right)^{s-1} V^{s}$.

Theorem 2. Suppose that system (4) has a PDS $(Y, Z)$. Then,

(i) $A^{*} A+B^{*} B<I$ and $C^{*} C+D^{*} D<I$

(ii) $Y^{-1}>A A^{*}, Y^{-1}>D D^{*}, Z^{-1}>C C^{*}$, and $Z^{-1}>B B^{*}$

(iii) $Y<\left(1 / s_{n}(A)^{2}\right) I$ if $A$ is nonsingular, $Y<\left(1 / s_{n}(D)^{2}\right) I$ if $D$ is nonsingular, $Z<\left(1 / s_{n}(C)^{2}\right) I$ if $C$ is nonsingular, and $Z<\left(1 / s_{n}(B)^{2}\right) I$ if $B$ is nonsingular

Proof. To prove (i), since $(Y, Z)$ is a PDS of the NMEs (4), we have $Y \geq I$ and $Z \geq I$. Together with (4), this gives

$$
A^{*} A+B^{*} B \leq A^{*} Y A+B^{*} Z B=I-Y^{-1}<I,
$$

and

$$
C^{*} C+D^{*} D \leq C^{*} Z C+D^{*} Y D=I-Z^{-1}<I .
$$

To prove (ii), from (6), we have

$$
\begin{aligned}
A^{*} Y A & <I, \\
A^{*} Y^{1 / 2} Y^{1 / 2} A & <I, \\
Y^{1 / 2} A A^{*} Y^{1 / 2} & <I, \\
A A^{*} & <Y^{-1}, \\
B^{*} Z B & <I, \\
B B^{*} & <Z^{-1} .
\end{aligned}
$$

Similarly, by (9), we can obtain $C C^{*}<Z^{-1}$ and $D D^{*}<Y^{-1}$. obtain

To prove (iii), if $A$ is nonsingular, then, by $A A^{*}<Y^{-1}$, we

$$
\left(A A^{*}\right)^{-1}>Y
$$

We have

$$
\frac{1}{s_{1}^{2}(A)} I \leq\left(A A^{*}\right)^{-1} \leq \frac{1}{s_{n}^{2}(A)} I .
$$

Since $s_{n}^{2}(A) I \leq A A^{*} \leq s_{1}^{2}(A) I$, where $s_{1}(A)$ and $s_{n}(A)$ are the maximal and the minimal singular value of the matrix $A$, respectively, by (11) and (12) and Remark 1, we obtain $Y<\left(A A^{*}\right)^{-1}<\left(1 / s_{n}(A)^{2}\right) I$.

For the case of nonsingular matrices $D, C$, and $B$, we can obtain similarly.

Theorem 3. System (4) attains a PDS $(Y, Z)$ iff $A, B, C, D$ admit the subsequent factorizations:

$$
\begin{aligned}
& A=\left(W^{*} W\right)^{-(1 / 2)} K_{1}, \\
& B=\left(U^{*} U\right)^{-(1 / 2)} K_{2}, \\
& C=\left(U^{*} U\right)^{-(1 / 2)} L_{1}, \\
& D=\left(W^{*} W\right)^{-(1 / 2)} L_{2},
\end{aligned}
$$


where the matrices $W$ and $U$ are nonsingular and the columns $\left(\begin{array}{c}W^{-*} \\ K_{1} \\ K_{2}\end{array}\right)$ and $\left(\begin{array}{c}U^{-*} \\ L_{1} \\ L_{2}\end{array}\right)$ are orthonormal. In this case, $(Y, Z)=\left(W^{*} W, U^{*} U\right)$ is a solution of $(4)$.
Proof. If the system of NMEs (4) has a PDS $(Y, Z)$, then $(Y, Z)=\left(W^{*} W, U^{*} U\right)$ for some nonsingular matrices $W, U$. Rewrite the equation (4) as

$$
\left\{\begin{array}{l}
\left(W^{*} W\right)^{-1}+A^{*}\left(W^{*} W\right) A+B^{*}\left(U^{*} U\right) B=I \\
\left(U^{*} U\right)^{-1}+C^{*}\left(U^{*} U\right) C+D^{*}\left(W^{*} W\right) D=I
\end{array}\right.
$$

or

$$
\left\{\begin{array}{l}
\left(W^{-*}\right)^{*} W^{-*}+\left[\left(W^{*} W\right)^{1 / 2} A\right]^{*}\left(W^{*} W\right)^{1 / 2} A+\left[\left(U^{*} U\right)^{1 / 2} B\right]^{*}\left(U^{*} U\right)^{1 / 2} B=I, \\
\left(U^{-*}\right)^{*} U^{-*}+\left[\left(U^{*} U\right)^{1 / 2} C\right]^{*}\left(U^{*} U\right)^{1 / 2} C+\left[\left(W^{*} W\right)^{1 / 2} D\right]^{*}\left(W^{*} W\right)^{1 / 2} D=I,
\end{array}\right.
$$

or equivalently

$$
\begin{aligned}
& \left(\begin{array}{c}
W^{-*} \\
\left(W^{*} W\right)^{1 / 2} A \\
\left(U^{*} U\right)^{1 / 2} B
\end{array}\right)^{*}\left(\begin{array}{c}
W^{-*} \\
\left(W^{*} W\right)^{1 / 2} A \\
\left(U^{*} U\right)^{1 / 2} B
\end{array}\right)=I \\
& \left(\begin{array}{c}
U^{-*} \\
\left(U^{*} U\right)^{1 / 2} C \\
\left(W^{*} W\right)^{1 / 2} D
\end{array}\right)^{*}\left(\begin{array}{c}
U^{-*} \\
\left(U^{*} U\right)^{1 / 2} C \\
\left(W^{*} W\right)^{1 / 2} D
\end{array}\right)=I .
\end{aligned}
$$

Let

$$
\begin{gathered}
\left(W^{*} W\right)^{1 / 2} A=K_{1}, \\
\left(U^{*} U\right)^{1 / 2} B=K_{2}, \\
\left(U^{*} U\right)^{1 / 2} C=L_{1}, \\
\left(W^{*} W\right)^{1 / 2} D=L_{2} .
\end{gathered}
$$

Then,

$$
\begin{aligned}
& A=\left(W^{*} W\right)^{-(1 / 2)} K_{1}, \\
& B=\left(U^{*} U\right)^{-(1 / 2)} K_{2}, \\
& C=\left(U^{*} U\right)^{-(1 / 2)} L_{1}, \\
& D=\left(W^{*} W\right)^{-(1 / 2)} L_{2} .
\end{aligned}
$$

Now, (16) implies that $\left(\begin{array}{c}W^{-*} \\ K_{1} \\ K_{2}\end{array}\right)$ and $\left(\begin{array}{c}U^{-*} \\ L_{1} \\ L_{2}\end{array}\right)$ are orthonormal. Conversely, assume that $A, B, C$, and $D$ have decomposition (13). Set $(Y, Z)=\left(W^{*} W, U^{*} U\right)$. Then,

$$
\begin{aligned}
Y^{-1}+A^{*} Y A+B^{*} Z B= & \left(W^{*} W\right)^{-1}+\left[\left(W^{*} W\right)^{-(1 / 2)} K_{1}\right]^{*}\left(W^{*} W\right)\left(W^{*} W\right)^{-(1 / 2)} K_{1} \\
& +\left[\left(U^{*} U\right)^{-(1 / 2)} K_{2}\right]^{*}\left(U^{*} U\left(U^{*} U\right)^{-(1 / 2)} K_{2}\right. \\
= & W^{-1} W^{-*}+K_{1}^{*} K_{1}+K_{2}^{*} K_{2}=I, \\
Z^{-1}+C^{*} Z C+D^{*} Y D= & \left(W^{*} W\right)^{-1}+\left[\left(U^{*} U\right)^{-(1 / 2)} L_{1}\right]^{*}\left(U^{*} U\right)\left(U^{*} U\right)^{-(1 / 2)} L_{1} \\
& +\left[\left(W^{*} W\right)^{-(1 / 2)} L_{2}\right]^{*}\left(W^{*} W\right)\left(W^{*} W\right)^{-(1 / 2)} L_{2} \\
= & U^{-1} U^{-*}+L_{1}^{*} L_{1}+L_{2}^{*} L_{2}=I,
\end{aligned}
$$

which implies $(Y, Z)=\left(W^{*} W, U^{*} U\right)$ is a PDS of (4). 
Example 1. We can find $W, U, K_{1}, K_{2}, L_{1}$, and $L_{2}$ (see Theorem 7.2.7, page 440, in [17]) for the following numerical experiment (2) which satisfies all the conditions of Theorem 3:

$$
\begin{aligned}
W & =\left[\begin{array}{lll}
1.0355 & 0.0341 & 0.0307 \\
0.0341 & 1.0448 & 0.0359 \\
0.0307 & 0.0359 & 1.0335
\end{array}\right], \\
U & =\left[\begin{array}{ccc}
1.0314 & -0.0004 & 0.0020 \\
-0.0004 & 1.0276 & 0.0003 \\
0.0020 & 0.0003 & 1.0489
\end{array}\right], \\
K_{1} & =\left[\begin{array}{ccc}
0.0446 & 0.1012 & 0.0750 \\
0.0667 & 0.1494 & 0.0809 \\
0.1614 & 0.1460 & 0.1117
\end{array}\right], \\
K_{2} & =\left[\begin{array}{ccc}
0.1087 & 0.0182 & 0.0888 \\
0.0770 & 0.1376 & 0.1556 \\
0.1089 & 0.0686 & 0.0387
\end{array}\right], \\
L_{1} & =\left[\begin{array}{ccc}
0.2442 & 0.0011 & 0.0017 \\
-0.0059 & 0.2283 & -0.0207 \\
0.0122 & 0.0224 & 0.2800
\end{array}\right], \\
L_{2}= & {\left[\begin{array}{ccc}
-0.0104 & 0.0009 & -0.0024 \\
-0.0014 & -0.0171 & -0.0143 \\
0.0043 & 0.0110 & -0.1093
\end{array}\right] . }
\end{aligned}
$$

Also, it is easy to derive

$$
\begin{aligned}
& s_{1}(A)=0.2981 \\
& s_{n}(A)=0.0146 \\
& s_{1}(B)=0.2691 \\
& s_{n}(B)=0.048 \\
& s_{1}(C)=0.2692 \\
& s_{n}(C)=0.2227 \\
& s_{1}(D)=0.1065 \\
& s_{n}(D)=0.0101
\end{aligned}
$$

It meets all of the requirements of Theorem 2 .

\section{Construction of Iteration Schemes}

This section contains a new iteration scheme for the NMEs (4) in the context of $[2,11]$.

By pre- and postmultiplying the first and the second equation of (4), respectively, by $Y$ and $Z$, we obtain

$$
\begin{aligned}
& Y-Y\left(I-A^{*} Y A-B^{*} Z_{2} B\right) Y=O \\
& Z-Z\left(I-C^{*} Z C-D^{*} Y D\right) Z=O .
\end{aligned}
$$

After some simple calculation, we have

$$
\begin{aligned}
& Y=2 Y-Y\left(I-A^{*} Y A-B^{*} Z B\right) Y, \\
& Z=2 Z-Z\left(I-C^{*} Z C-D^{*} Y D\right) Z .
\end{aligned}
$$

Apparently, $(Y, Z)$ is a solution of $(23)$ if $(Y, Z)$ solves equation (4). Conversely, if $(Y, Z)$ is a nonsingular solution of equation (23), $(Y, Z)$ solves equation (4), too.

Thus, to attain a HPDS of (4), we need to solve (23). From (23), the iterative scheme is as follows.

$$
\left\{\begin{array}{l}
\widehat{Y}_{n}=I-A^{*} Y_{n} A-B^{*} Z_{n} B \\
Y_{n+1}=\left(2 I-Y_{n} \widehat{Y}_{n}\right) Y_{n}, \\
\widehat{Z}_{n}=I-C^{*} Z_{n} C-D^{*} Y_{n+1} D \\
Z_{n+1}=\left(2 I-Z_{n} \widehat{Z}_{n}\right) Z_{n} .
\end{array}\right.
$$

\section{Convergence Analysis}

This section contains the proof that the sequences $\left\{\left(Y_{n}, Z_{n}\right)\right\}$ generated by Algorithm 1, where $Y_{0}=I$ and $Z_{0}=I$ are initial conditions and converge to the minimal positivedefinite solution of equation (4).

Theorem 4. Suppose that the system of NMEs (4) has a PDS and the sequence $\left\{\left(Y_{n}, Z_{n}\right)\right\}$ is generated by Algorithm 1 with initial values $Y_{0}=I$ and $Z_{0}=I$. Let the pair $(\widehat{Y}, \widehat{Z})$ be the minimal PDS of (4). Then, the sequence $\left\{\left(Y_{n}, Z_{n}\right)\right\}$ is well defined:

$$
\begin{aligned}
Y_{0} & \leq Y_{1} \leq Y_{2} \leq \cdots \leq Y_{n} \leq \cdots \leq \widehat{Y}, \\
Z_{0} & \leq Z_{1} \leq Z_{2} \leq \cdots<Z_{n} \leq \cdots \leq \widehat{Z}, \\
\lim _{n \longrightarrow \infty} Y_{n} & =\widehat{Y}, \\
\lim _{n \rightarrow \infty} Z_{n} & =\widehat{Z} .
\end{aligned}
$$

Proof. Let $(Y, Z)$ be any PDS of the system of NMEs (4). We will prove that $O<Y_{n} \leq Y_{n+1} \leq Y$ and $O<Z_{n} \leq Z_{n+1} \leq Z$ for all $n \in \mathbb{N}^{*}=\mathbb{N} \cup\{0\}$ using mathematical induction.

For $n=0$, we have $Y_{0}=I>0$ and $Z_{0}=I>O$. Then, by (24), we have

$$
\begin{aligned}
& Y_{1}=2 I-I+A^{*} A+B^{*} B=I+A^{*} A+B^{*} B \geq I=Y_{0}, \\
& Z_{1}=2 I-I+C^{*} C+D^{*} Y_{1} D=I+C^{*} C+D^{*} Y_{1} D \geq I=Z_{0} .
\end{aligned}
$$

Following Theorem 4, $I-A^{*} A-B^{*} B>0$. Using this condition in Lemma 1 together with NMEs (4),

$$
\begin{aligned}
Y_{1} & =2 I-I\left(I-A^{*} A-B^{*} B\right) I \\
& \leq\left(I-A^{*} A-B^{*} B\right)^{-1} \\
& \leq\left(I-A^{*} Y A-B^{*} Z B\right)^{-1} \\
& =Y,
\end{aligned}
$$


Step 1: input $A, B, C, D \in \mathbb{C}^{n \times n}$.

Take $Y_{0}=I$ and $Z_{0}=I$ for initialization and tolerance error $\epsilon \geq 0$. Set $n$ : $=0$.

Step 2: find out $Y_{n+1}$ and $Z_{n+1}$ by the following iterative scheme:

Step 3: stop if $\left\|Y_{n+1}^{n+1}-Y_{n}\right\|_{F}+\left\|Z_{n+1}-Z_{n}\right\|_{F} \leq \epsilon$. Otherwise, $n$ : $=n+1$, and go to Step 2 .

Algorithm 1: An inversion-free iterative algorithm for equation (4).

since $(Y, Z) \geq(I, I)$. That is, $Y_{1} \leq Y$. Using Lemma 2 , this implies that $I-C^{*} C-D^{*} Y_{1} D \geq I-C^{*} Z C-D^{*} Y D=$ $Z^{-1}>0$. Using this fact in Lemma 1 together with NMEs (4), we obtain

$$
\begin{aligned}
Z_{1} & =2 I-I\left(I-C^{*} C-D^{*} Y_{1} D\right) I \\
& \leq\left(I-C^{*} C-D^{*} Y_{1} D\right)^{-1} \\
& \leq\left(I-C^{*} Z C-D^{*} Y D\right)^{-1} \\
& =Z .
\end{aligned}
$$

Hence, $O<Y_{n} \leq Y_{n+1} \leq Y$ and $O<Z_{n} \leq Z_{n+1} \leq Z$ holds true for $n=0$.

Assume that $O<Y_{n} \leq Y_{n+1} \leq Y$ and $O<Z_{n} \leq Z_{n+1} \leq Z$ hold for $n=k \geq 0$. Since $I-A^{*} Y_{k+1} A-B^{*} Z_{k+1} B \geq$ $I-A^{*} Y A-B^{*} Z B=Y^{-1}>O$, by equation (24), Lemma 1, and the fact $Y_{k+1}>O$, we obtain

$$
\begin{aligned}
Y_{k+2} & =2 Y_{k+1}-Y_{k+1}\left(I-A^{*} Y_{k+1} A-B^{*} Z_{k+1} B\right) Y_{k+1} \\
& \leq\left(I-A^{*} Y_{k+1} A-B^{*} Z_{k+1} B\right)^{-1} \\
& \leq\left(I-A^{*} Y A+B^{*} Z B\right)^{-1} \\
& =Y,
\end{aligned}
$$

that is, $Y_{k+2} \leq Y$. Then, by this condition and Lemma 2, we have

$I-C^{*} Z_{k+1} C+D^{*} Y_{k+2} D \geq I-C^{*} Z C-D^{*} Y D=Z^{-1}>0$.

Using this fact in Lemma 1 together with the NMEs (22) and the fact $Z_{i+1}>O$, we have

$$
\begin{aligned}
Z_{k+2} & =2 Z_{k+1}-Z_{k+1}\left(I-C^{*} Z_{k+1} C-D^{*} Y_{k+2} D\right) Z_{k+1} \\
& \leq\left(I-C^{*} Z_{k+1} C-D^{*} Y_{k+2} D\right)^{-1} \\
& \leq Z
\end{aligned}
$$

Also, from the NMEs (24), we get that

$$
\begin{aligned}
Y_{k+2}-Y_{k+1} & =Y_{k+1}-Y_{k+1}\left(I-A^{*} Y_{k+1} A-B^{*} Z_{k+1} B\right) Y_{k+1} \\
& =Y_{k+1}\left[Y_{k+1}^{-1}-\left(I-A^{*} Y_{k+1} A-B^{*} Z_{k+1} B\right)\right] Y_{k+1}
\end{aligned}
$$

Next, by using the fact $I-A^{*} Y_{k} A-B^{*} Z_{k} B \geq$ $I-A^{*} Y_{k+1} A-B^{*} Z_{k+1} B>O$ together with the NMEs (24), Lemma 1 , and the fact $Y_{k}>0$, it follows that

$$
\begin{aligned}
Y_{k+1} & =2 Y_{k}-Y_{k}\left(I-A^{*} Y_{k} A-B^{*} Z_{k} B\right) Y_{k} \\
& \leq\left(I-A^{*} Y_{k} A-B^{*} Z_{k} B\right)^{-1} \\
& \leq\left(I-A^{*} Y_{k+1} A-B^{*} Z_{k+1} B\right)^{-1},
\end{aligned}
$$

that is, $Y_{k+1} \leq\left(I-A^{*} Y_{k+1} A-B^{*} Z_{k+1} B\right)^{-1}$ and $Y_{k+1}^{-1} \geq$ $I-A^{*} Y_{k+1} A-B^{*} Z_{k+1} B$. Using this fact with $Y_{k+1}>O$, in equation (31), we have $Y_{k+2} \geq Y_{k+1}$. Using this fact with Lemma 2, we have

$$
I-C^{*} Z_{k} C-D^{*} Y_{k+1} D \geq I-C^{*} Z_{k+1} C-D^{*} Y_{k+2} D>O \text {. }
$$

This fact, together with the NMEs (24), Lemma 1, and the fact $Z_{k}>O$, yields

$$
\begin{aligned}
Z_{k+1} & =2 Z_{k}-Z_{k}\left(I-C^{*} Z_{k} C-D^{*} Y_{k+1} D\right) Z_{k} \\
& \leq\left(I-C^{*} Z_{k} C-D^{*} Y_{k+1} D\right)^{-1} \\
& \leq\left(I-C^{*} Z_{k+1} C-D^{*} Y_{k+2} D\right)^{-1},
\end{aligned}
$$

that is, $Z_{k+1} \leq\left(I-C^{*} Z_{k+1} C-D^{*} Y_{k+2} D\right)^{-1}$ or $Z_{k+1}^{-1} \geq I-$ $C^{*} Z_{k+1} C-D^{*} Y_{k+2} D$. Thereby,

$$
\begin{aligned}
Z_{k+2}-Z_{k+1} & =Z_{k+1}-Z_{k+1}\left(I-C^{*} Z_{k+1} C-D^{*} Y_{k+2} D\right) Z_{k+1} \\
& =Z_{k+1}\left[Z_{k+1}^{-1}-\left(I-C^{*} Z_{k+1} C-D^{*} Y_{k+2} D\right)\right] Z_{k+1} \geq O .
\end{aligned}
$$

From the above, $O<Y_{n} \leq Y_{n+1} \leq Y$ and $O<Z_{n} \leq Z_{n+1} \leq Z$ hold for $n=k+1$.

Thus, by using the principle of induction, we have that the relations $O<Y_{n} \leq Y_{n+1} \leq Y$ and $O<Z_{n} \leq Z_{n+1} \leq Z$ hold for $n \in \mathbb{N}^{*}$. So, the sequence $\left\{\left(Y_{n}, Z_{n}\right)\right\}$ is well defined, increasing, and bounded above. Let

$$
\begin{aligned}
& \lim _{n \longrightarrow \infty} Y_{n}=\widehat{Y}, \\
& \lim _{n \longrightarrow \infty} Z_{n}=\widehat{Z} .
\end{aligned}
$$

Then, $(\widehat{Y}, \widehat{Z})$ is a PDS of the NMEs (4) by Algorithm 1 . Since $(\widehat{Y}, \widehat{Z}) \leq(Y, Z)$ for any $\operatorname{PDS}(Y, Z)$ of NMEs (4), $(\widehat{Y}, \widehat{Z})$ is the minimal PSD of NMEs $(4)$.

Remark 2. If $\left(\widehat{X}_{1}, \widehat{X}_{2}\right)$ is the maximal PDS of the system of NMEs (3), then by the relationship between the NMEs (3) and (4), we get that $\left(\widehat{X}_{1}^{-1}, \widehat{X}_{2}^{-1}\right)$ is the minimal PDS of NMEs (4). Therefore, from Theorem 4 , the sequence $\left\{\left(Y_{n}, Z_{n}\right)\right\}$ generated by Algorithm 1 with the initialization $Y_{0}=I$ and $Z_{0}=I$ converges to the inverse of the maximal PDS of NMEs (3), respectively. 


\section{Rate of Convergence}

Lemma 4 (see [18]). If $0<\theta \leq 1$ and $U, V \in P(n)$ with $U, V \geq c I>O$, then, for every unitarily invariant norm, $\left\|\left|U^{\theta}-V^{\theta}\left\|\left|\leq \theta c^{\theta-1}\||U-V \||\right.\right.\right.\right.$ and $\left.\left.\left.\|\right| U^{-\theta}-V^{-\theta}\right\|\right| \leq \theta c^{-(\theta+1)}$ $\||U-V \||$

Theorem 5. If $(Y, Z)$ is a PDS of the matrix equation (4) under Algorithm 1 and $\epsilon>0$ is arbitrary, then we get the following.

(A)

$$
\left\|Y_{n}-Y\right\| \leq(\|A Y\|+\varepsilon)^{2}\left\|Y-Y_{n}\right\|+(\|B Y\|+\varepsilon)^{2}\left\|Z-Z_{n}\right\|,
$$

$$
\left\|\widehat{Y}_{n}-Y^{-1}\right\| \leq\|A\|^{2}\left\|Y_{n}-Y\right\|+\|B\|^{2}\left\|Z_{n}-Z\right\| .
$$

(B)

$\left\|Z_{n}-Z\right\| \leq(\|C Z\|+\varepsilon)^{2}\left\|Z_{n}-Z\right\|+(\|D Z\|+\varepsilon)^{2}\left\|Y_{n+1}-Y\right\|$,

and

$$
\left\|\widehat{Z}_{n}-Z^{-1}\right\| \leq\|C\|^{2}\left\|Z_{n}-Z\right\|+\|D\|^{2}\left\|Y_{n+1}-Y\right\|,
$$

for all $n$ large enough.

Proof. To prove (A), let $(Y, Z)$ be the PDS of equation (4). Then, from Algorithm 1,

and

$$
\begin{aligned}
Y_{n+1} & =2 Y_{n}-Y_{n}\left(I-A^{*} Y_{n} A-B^{*} Z_{n} B\right) Y_{n} \\
& =2 Y_{n}-Y_{n}\left[I-A^{*}\left(Y+Y_{n}-Y\right) A-B^{*}\left(Z+Z_{n}-Z\right) B\right] Y_{n} \\
& =2 Y_{n}-Y_{n}\left[I-A^{*} Y A-B^{*} Z B+A^{*}\left(Y-Y_{n}\right) A+B^{*}\left(Z-Z_{n}\right) B\right] Y_{n} \\
& =2 Y_{n}-Y_{n} Y^{-1} Y_{n}-Y_{n} A^{*}\left(Y-Y_{n}\right) A Y_{n}-Y_{n} B^{*}\left(Z-Z_{n}\right) B Y_{n} . \\
& X_{1(k+1)}=I-A^{*} X_{1(k)}^{-1} A-B^{*} X_{2(k)}^{-1} B, \\
-2 Y_{n}+Y_{n} Y^{-1} Y_{n}=\left(Y-Y_{n}\right) Y^{-1} & X_{2(k+1)}=I-C^{*} X_{2(k)}^{-1} C-D^{*} X_{1(k)}^{-1} D .
\end{aligned}
$$

Thus, since $\quad Y-2 Y_{n}+Y_{n} Y^{-1} Y_{n}=\left(Y-Y_{n}\right) Y^{-1}$ $\left(Y-Y_{n}\right)$, we have

$$
\begin{aligned}
Y-Y_{n+1}= & \left(Y-Y_{n}\right) Y^{-1}\left(Y-Y_{n}\right)+Y_{n} A^{*}\left(Y-Y_{n}\right) A Y_{n} \\
& +Y_{n} B^{*}\left(Z-Z_{n}\right) B Y_{n} .
\end{aligned}
$$

Now, since $\lim _{n \rightarrow \infty} Y_{n}=Y$, using Lemma 4, we have

$$
\begin{aligned}
\left\|Y-Y_{n+1}\right\| \leq & \left\|\left(Y-Y_{n}\right) Y^{-1}\right\|\left\|Y-Y_{n}\right\|+\left\|A Y_{n}\right\|^{2}\left\|Y-Y_{n}\right\| \\
& +\left\|B Y_{n}\right\|\left\|Z-Z_{n}\right\| \\
\leq & {\left[\left\|\left(Y-Y_{n}\right) Y^{-1}\right\|+\left\|A Y_{n}\right\|^{2}\right]\left\|Y-Y_{n}\right\|+\left\|B Y_{n}\right\|^{2} } \\
& \left\|Z-Z_{n}\right\| \\
\leq & (\|A Y\|+\varepsilon)^{2}\left\|Y-Y_{n}\right\|+(\|B Y\|+\varepsilon)^{2}\left\|Z-Z_{n}\right\| .
\end{aligned}
$$

which is (35).

Since

$$
\widehat{Y}_{n}-Y^{-1}=A^{*}\left(Y-Y_{n}\right) A+B^{*}\left(Z-Z_{n}\right) B,
$$

using Lemma 4, we have relation (38).

In the same way, we can prove (B).

\section{Numerical Examples}

Two examples are presented in this section in support of Algorithm 1. Take Residue $=\left\|Y_{n+1}-Y_{n}\right\|+\left\|Z_{n+1}-Z_{n}\right\|$, tolerance $=1 e-10$, and norm as Frobenius norm. For comparative analysis, a basic fixed point algorithm (BFP, for short) has been used:
These examples, together with tables and graphs showing different input data, solutions, iteration number of different schemes, error, CPU time, average computational time, etc., are illustrated here. For a better understanding, we have used line graphs, bar graphs, pie graphs, and surface plotting.

Example 2. Take

$$
\begin{aligned}
A & =\left[\begin{array}{lll}
0.0367 & 0.0893 & 0.0670 \\
0.0574 & 0.1355 & 0.0717 \\
0.1530 & 0.1339 & 0.1036
\end{array}\right], \\
B & =\left[\begin{array}{lll}
0.1052 & 0.0175 & 0.0861 \\
0.0749 & 0.1339 & 0.1514 \\
0.1036 & 0.0654 & 0.0367
\end{array}\right], \\
C & =\left[\begin{array}{ccc}
0.2367 & 0.0011 & 0.0011 \\
-0.0056 & 0.2221 & -0.0202 \\
0.0112 & 0.0213 & 0.2670
\end{array}\right], \\
D & =\left[\begin{array}{ccc}
-0.0101 & 0.0011 & 0.0011 \\
-0.0011 & -0.0168 & -0.0101 \\
0.0045 & 0.0112 & -0.1055
\end{array}\right] .
\end{aligned}
$$

After applying Algorithm 1 with the initial conditions $Y_{0}=I$ and $Z_{0}=I$, we obtain $X_{1}=$ $\left[\begin{array}{lll}0.9379 & -0.0581 & -0.0526\end{array}\right]$ and $X_{2}=\left[\begin{array}{lll}0.9401 & 0.0007 & -0.0035 \\ 0.007 & 0.9470 & -0.0006\end{array}\right]$ $\left[\begin{array}{ccc}-0.0581 & 0.9221 & -0.0617 \\ -0.0526 & -0.0617 & 0.9418\end{array}\right]$ and $X_{2}=\left[\begin{array}{ccc}0.9401 & 0.0007 & -0.0035 \\ 0.0007 & 0.9470 & -0.0006 \\ -0.0035 & -0.0006 & 0.9090\end{array}\right]$. 
Table 1 presents a comparison between our algorithm and the basic fixed point algorithm.

Figures 1 and 2 represent CPU time graph and iteration vs. error graph.

Figures 3 and 4 represent pie chart for average CPU time based on ten experiments and solution's surface plot.

Example 3. In the current example, a new, randomly chosen set of coefficients $A, B, C, D \in \mathbb{C}^{n \times n}$ is used. The construction is followed by

$$
\begin{aligned}
A_{t}(i, j) & =\frac{r}{i+j-1}, \\
B_{t}(i, j) & =\frac{r}{1} \times A_{t}(i, j), \\
C_{t}(i, j) & =\frac{r^{2}}{i+j-1}, \\
D_{t}(i, j) & =\frac{1}{r-1} \times A_{t}(i, j), \\
Q_{1}(i, j) & =I(i, j)+A_{t}^{*}(i, j) \times A_{t}(i, j), \\
Q_{2}(i, j) & =I(i, j)+B_{t}^{*}(i, j) \times B_{t}(i, j), \\
A_{1}(i, j) & =Q_{1}(i, j)^{-(1 / 2)} \times A_{t}(i, j) \times Q_{1}(i, j)^{-(1 / 2)}, \\
B_{1}(i, j) & =Q_{1}(i, j)^{-(1 / 2)} \times B_{t}(i, j) \times Q_{1}(i, j)^{-(1 / 2)}, \\
C_{1}(i, j) & =Q_{2}(i, j)^{-(1 / 2)} \times C_{t}(i, j) \times Q_{2}(i, j)^{-(1 / 2)}, \\
D_{1}(i, j) & =Q_{2}(i, j)^{-(1 / 2)} \times D_{t}(i, j) \times Q_{2}(i, j)^{-(1 / 2)}, \\
N_{1} & =\operatorname{norm}\left(A_{1}^{*} \times A_{1}+B_{1}^{*} \times B_{1}, f r o^{\prime}\right), \\
N_{2} & =\operatorname{norm}\left(C_{1}^{*} \times C_{1}+D_{1}^{*} \times D_{1}, f r o^{\prime}\right), \\
A & =\frac{1}{r} \times\left(\frac{A_{1}}{N_{1}}+i \frac{B_{1}}{N_{1}}\right), \\
B & =\frac{1}{r} \times\left(\frac{B_{1}}{N_{1}}+i \frac{A_{1}}{N_{1}}\right), \\
C & =\frac{1}{r} \times\left(\frac{C_{1}}{N_{2}}+i \frac{D_{1}}{N_{1}}\right), \\
D & =\frac{1}{r} \times\left(\frac{C_{1}}{N_{2}}+i \frac{D_{1}}{N_{1}}\right) .
\end{aligned}
$$

Here, " $r$ " and "fro" stand for dimension and Frobenius norm of the matrix, respectively. A different set of initial conditions has been chosen here as

$$
\begin{aligned}
& Y_{0}=\left(\frac{1}{2}\right) \times A \times \text { conjugate-transpose }(A), \\
& Z_{0}=\left(\frac{1}{2}\right) \times B \times \text { conjugate-transpose }(B) .
\end{aligned}
$$

The result of this experiment using Algorithm 1 is shown in Table 2.

Example 4. Here, we consider some randomly generated matrices:

$$
\begin{aligned}
A_{1} & =I+\frac{1}{(2 \times r) \operatorname{rand}(r)}, \\
B_{1} & =I+\frac{1}{(r)^{2} \text { rand }(r)}, \\
C_{1} & =I+\frac{1}{(r)^{3} \text { rand }(r)}, \\
D_{1} & =I+\frac{1}{(r \times 3) \operatorname{rand}(r)}, \\
A & =\frac{A_{1}}{\left\|A_{1}\right\|}, \\
B & =\frac{B_{1}}{\left\|B_{1}\right\|}, \\
C & =\frac{C_{1}}{\left\|C_{1}\right\|}, \\
D & =\frac{D_{1}}{\left\|D_{1}\right\|},
\end{aligned}
$$

where $r=$ dimension of the matrices, $\operatorname{rand}(r)=$ random matrices of order $r$, and tol $=1 e-10$. After applying Algorithm 1 (ALgo1) and basic fixed-point method under the initial conditions $Y_{0}=I$ and $Z_{0}=I$, we get Table 3 that shows the various outputs for different $r$. The 5 th column of Table 3 ensures positive definiteness of Example 4 w.r.t. different order matrices.

Associated graphs are (Figures 5-25)
(i) $\operatorname{dim}=3\left\{\begin{array}{l}\text { Fig 5: CPU time } \\ \text { Fig 6: it. no. vs. error } \\ \text { Fig 7: Sols surface plot' }\end{array}\right.$
(ii) $\operatorname{dim}=5\left\{\begin{array}{l}\text { Fig 8: CPU time } \\ \text { Fig 9: it. no. vs. error } \\ \text { Fig 10: Sols surface plot' }\end{array}\right.$
(iii) $\operatorname{dim}=8\left\{\begin{array}{l}\text { Fig 11: CPU time } \\ \text { Fig 12: it. no. vs. error } \\ \text { Fig 13: Sols surface plot' }\end{array}\right.$
(iv) $\operatorname{dim}=12\left\{\begin{array}{l}\text { Fig 14: CPU time } \\ \text { Fig 15: it. no. vs. error } \\ \text { Fig 16: Sols surface plot }\end{array}\right.$ 
TABle 1: Comparison of Algorithm 1 with basic fixed point iteration.

\begin{tabular}{llccc}
\hline Method & $r$ & No. of iteration & Error & Average CPU time \\
\hline Algo1 & 3 & 12 & $0.5908 e-10$ & 0.0070 \\
BFP & 3 & 12 & $0.2180 e-10$ & 0.0157 \\
\hline
\end{tabular}

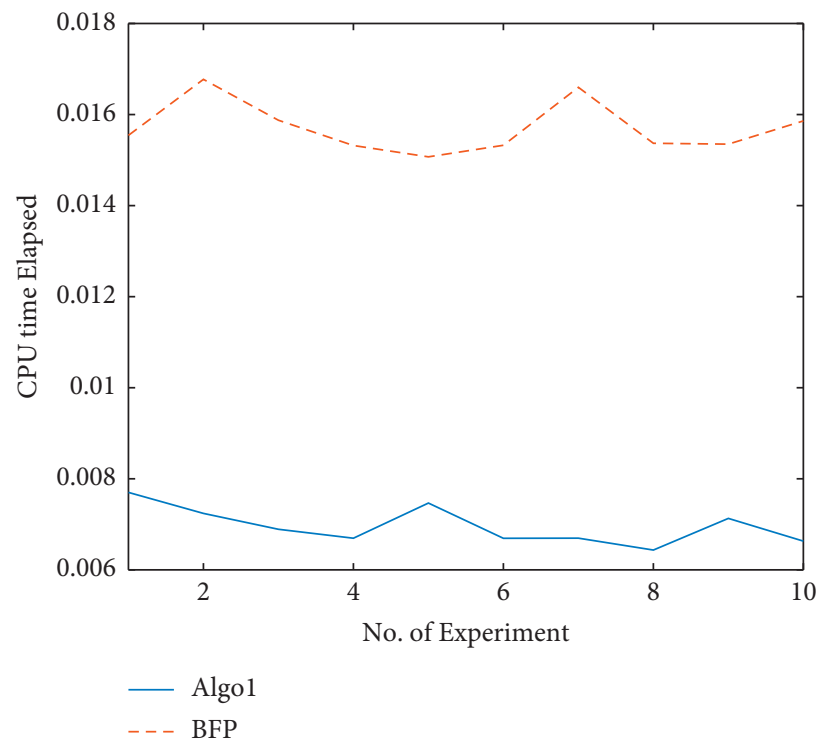

FIgURE 1: CPU time graph and iteration vs error graph.

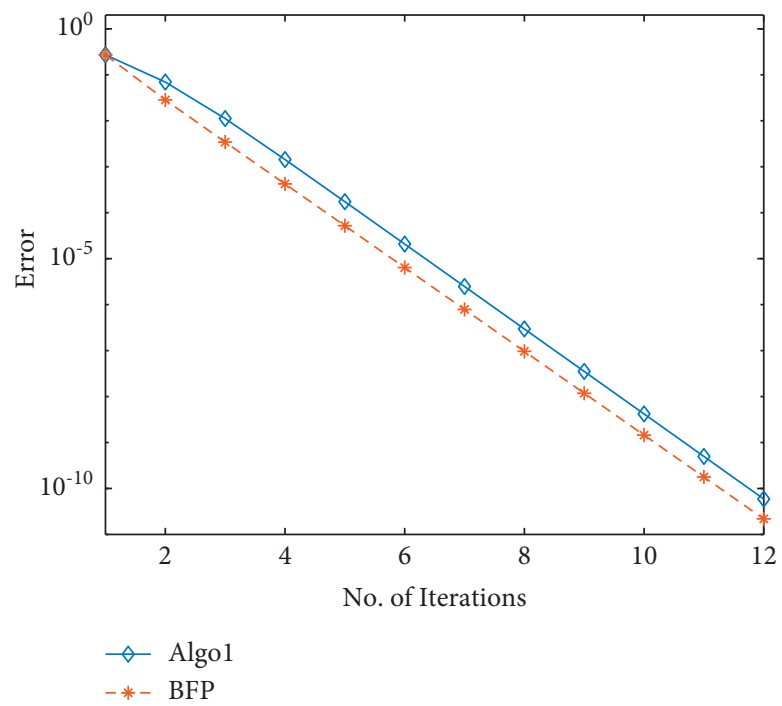

FIgURE 2: CPU time graph and iteration vs error graph.

(v) $\operatorname{dim}=20\left\{\begin{array}{l}\text { Fig 17: } \text { CPU time } \\ \text { Fig 18: it. no. vs. error } \\ \text { Fig 19: }\end{array}\right.$ Fig 19: Sols surface plot'

$\int$ Fig 20: CPU time

(vi) $\operatorname{dim}=32\{$ Fig 21: it. no. vs. error Fig 22: Sols surface plot'

(vii) $\operatorname{dim}=64\left\{\begin{array}{l}\text { Fig 23: CPU time } \\ \text { Fig 24: it. no. vs. error } \\ \text { Fig 25: Sols surface plot }\end{array}\right.$ (viii) Figure 26 represents the average CPU time through bar graphs for different dimensions

Remark 3. We can infer from the above discussions that our algorithm is less expensive in terms of computation after evaluating these examples w.r.t. different sets of parameters.

Remark 4. We can infer from the above discussions that the solutions are positive definite. 


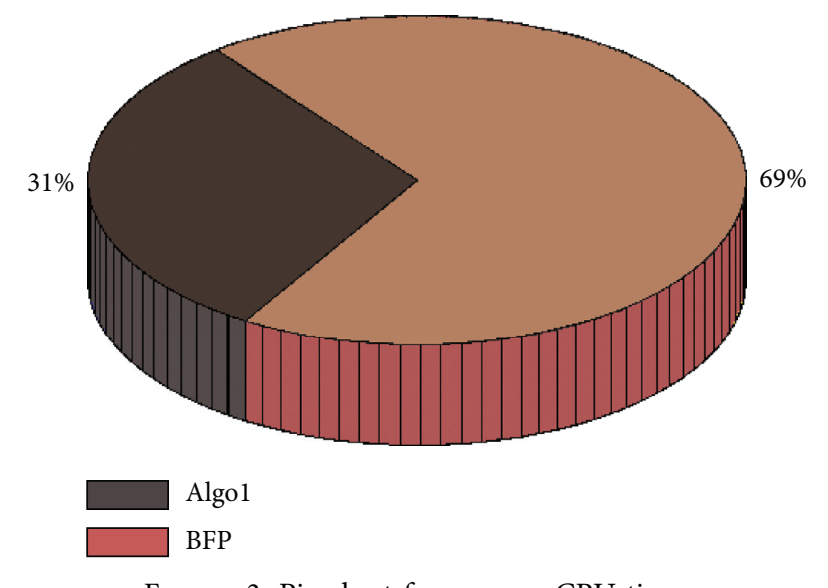

Figure 3: Pie chart for average CPU time.
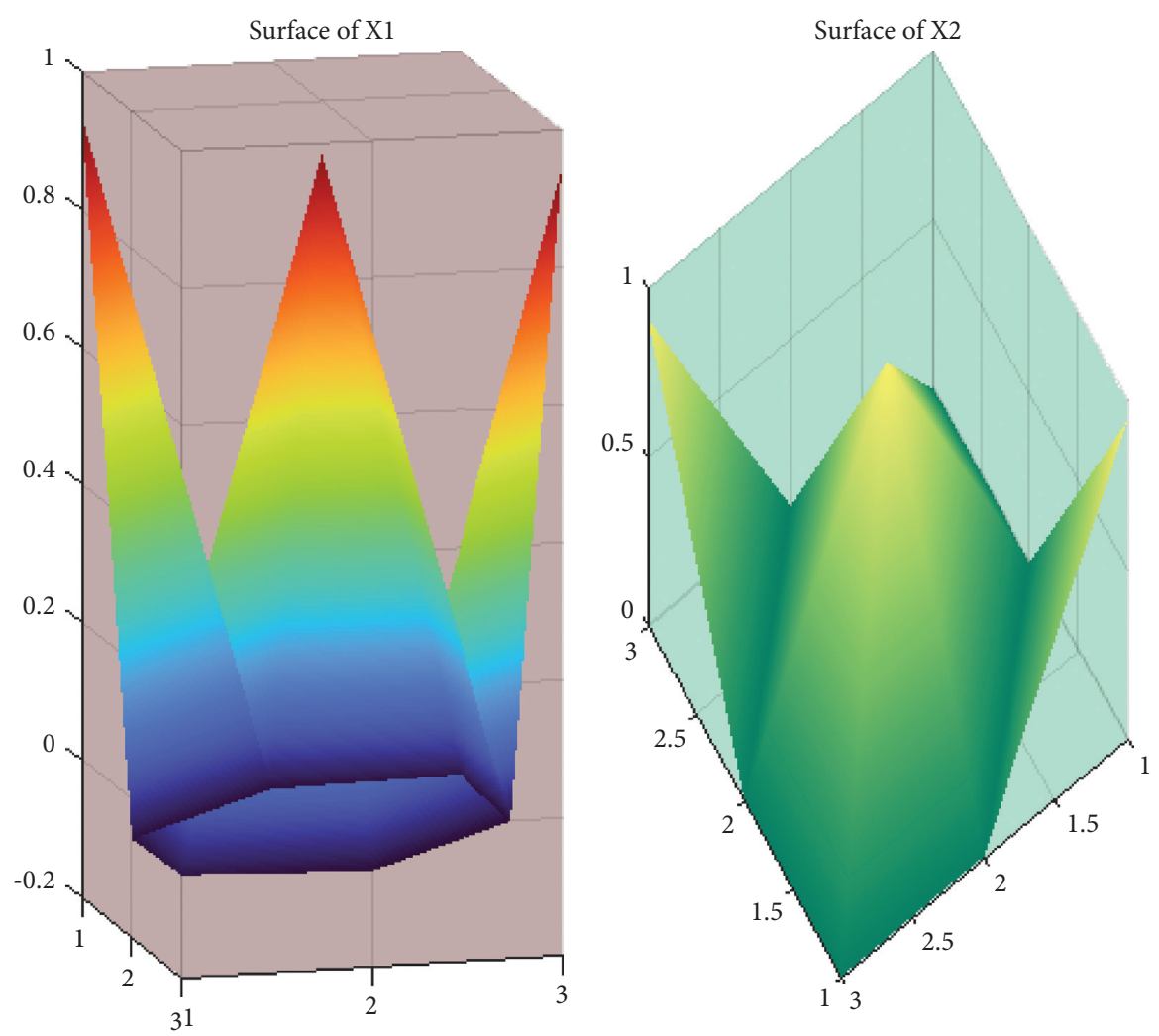

FIgURE 4: Solution surface plot. 
TABLE 2: CPU analysis for different dimension.

\begin{tabular}{lccc}
\hline Dim & No. of iteration & Error & CPU time (seconds) \\
\hline 3 & 8 & $3.0319 e-12$ & 0.007770 \\
4 & 6 & $6.4760 e-12$ & 0.006651 \\
5 & 5 & $2.2293 e-11$ & 0.008359 \\
10 & 4 & $2.0735 e-12$ & 0.007447 \\
200 & 3 & $7.9969 e-15$ & 0.030397 \\
500 & 2 & $1.1787 e-11$ & 0.111352 \\
1000 & 2 & $7.3933 e-13$ & 0.758877 \\
\hline
\end{tabular}

TABle 3: Comparison of Algorithm 1 with basic fixed point iteration for different dimension.

\begin{tabular}{|c|c|c|c|c|}
\hline Dim & & Algo 1 & BFP & $\operatorname{Min}\left(\right.$ eig) $X_{1}, X_{2}$ \\
\hline \multirow{3}{*}{3} & No. of iter. & 17 & 19 & \multirow{3}{*}{$0.7646,0.7620$} \\
\hline & Error $\left(\times 10^{-10}\right)$ & 0.6836 & 0.48240 & \\
\hline & Av. CPU time & 0.0064 & 0.0098 & \\
\hline \multirow{3}{*}{5} & No. of iter. & 11 & 13 & \multirow{3}{*}{$0.8689,0.8729$} \\
\hline & Error $\left(\times 10^{-10}\right)$ & 0.1686 & 0.3111 & \\
\hline & Av. CPU time & 0.0062 & 0.0096 & \\
\hline \multirow{3}{*}{8} & No. of iter. & 9 & 10 & \multirow{3}{*}{$0.9175,0.9238$} \\
\hline & Error $\left(\times 10^{-10}\right)$ & 0.8159 & 0.48613 & \\
\hline & Av. CPU time & 0.0072 & 0.0101 & \\
\hline \multirow{3}{*}{12} & No. of iter. & 8 & 9 & \multirow{3}{*}{$0.9450,0.9497$} \\
\hline & Error $\left(\times 10^{-10}\right)$ & 0.3285 & 0.0948 & \\
\hline & Av. CPU time & 0.0073 & 0.0104 & \\
\hline \multirow{3}{*}{20} & No. of iter. & 7 & 7 & \multirow{3}{*}{$0.9672,0.9701$} \\
\hline & Error $\left(\times 10^{-10}\right)$ & 0.2388 & 0.9457 & \\
\hline & Av. CPU time & 0.0077 & 0.0109 & \\
\hline \multirow{3}{*}{32} & No. of iter. & 6 & 7 & \multirow{3}{*}{$0.9796,0.9814$} \\
\hline & Error $\left(\times 10^{-10}\right)$ & 0.7179 & 0.0356 & \\
\hline & Av. CPU time & 0.0078 & 0.0116 & \\
\hline \multirow{3}{*}{64} & No. of iter. & 6 & 6 & \multirow{3}{*}{$0.9899,0.9907$} \\
\hline & Error $\left(\times 10^{-10}\right)$ & 0.1332 & 0.48082 & \\
\hline & Av. CPU time & 0.0124 & 0.0168 & \\
\hline
\end{tabular}

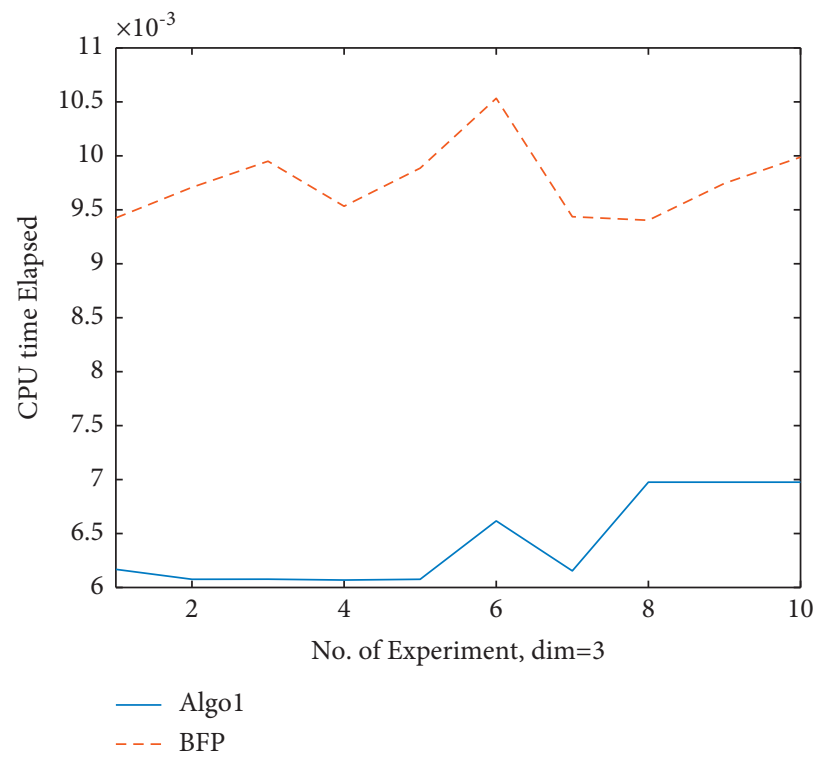

Figure 5: CPU time vs no. of iteration for dimension 3. 


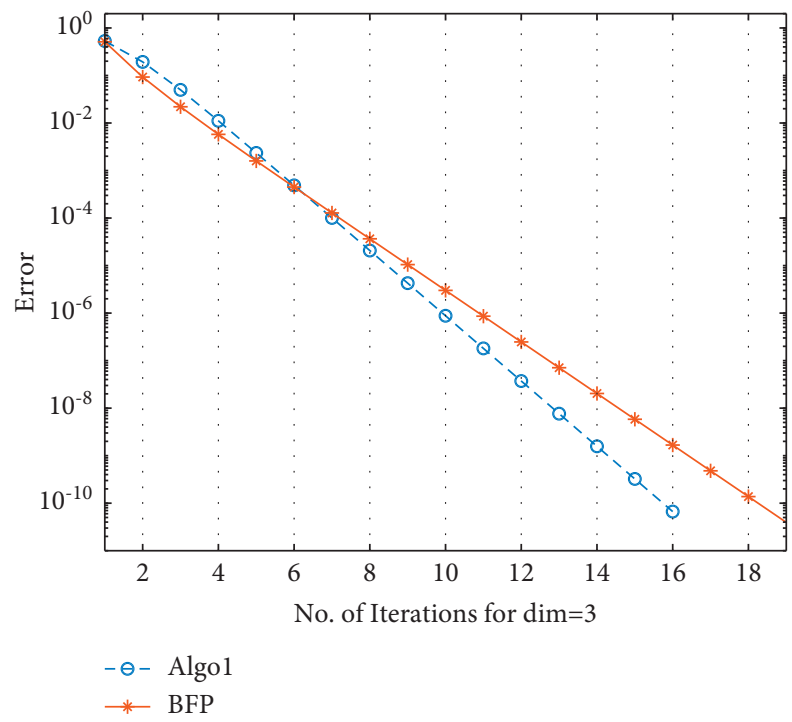

FIGURE 6: Iteration no. vs error for dimension 3.
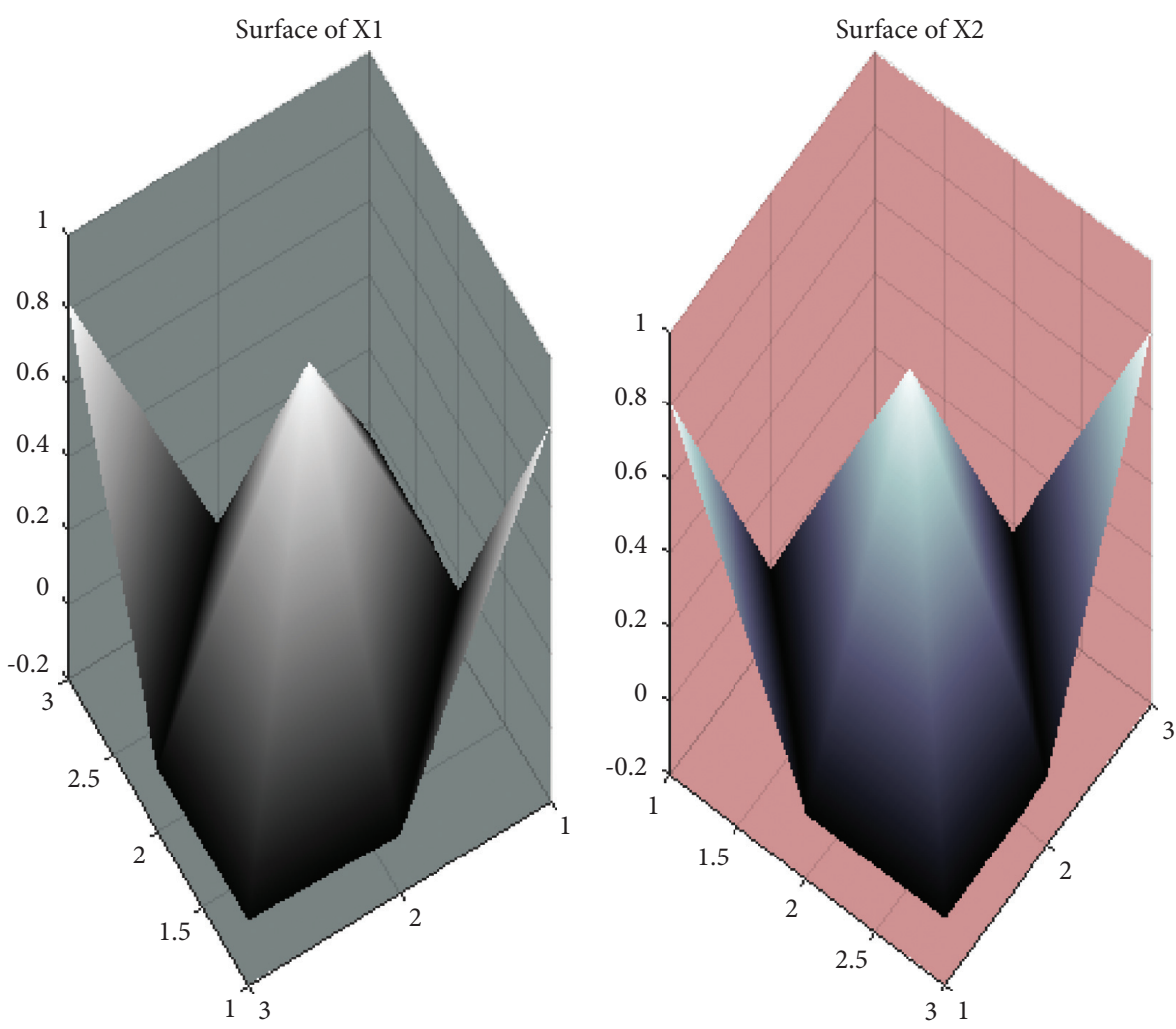

Figure 7: Solution surface plot for dimension 3. 


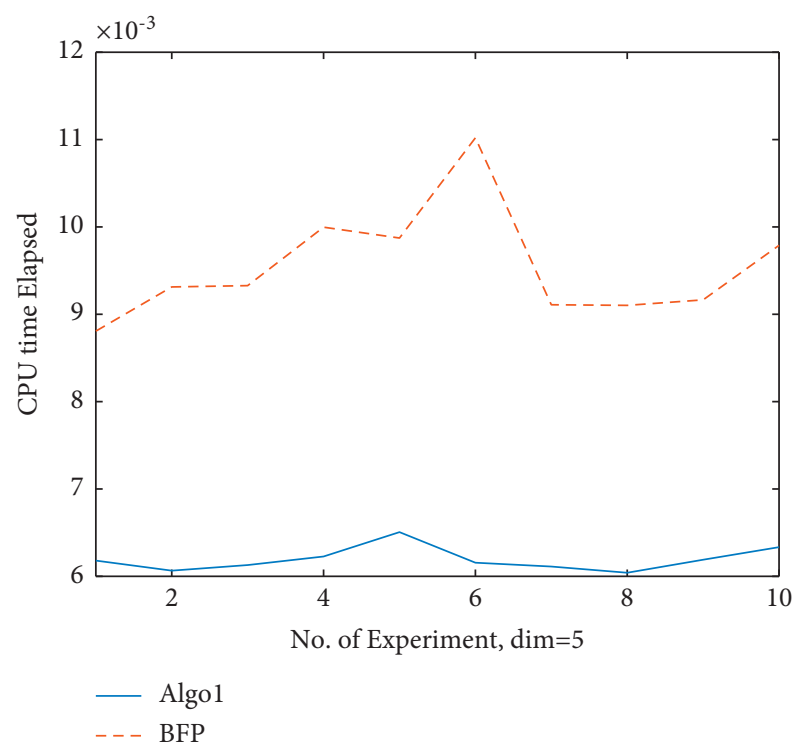

Figure 8: CPU time vs no. of iteration for dimension 5.

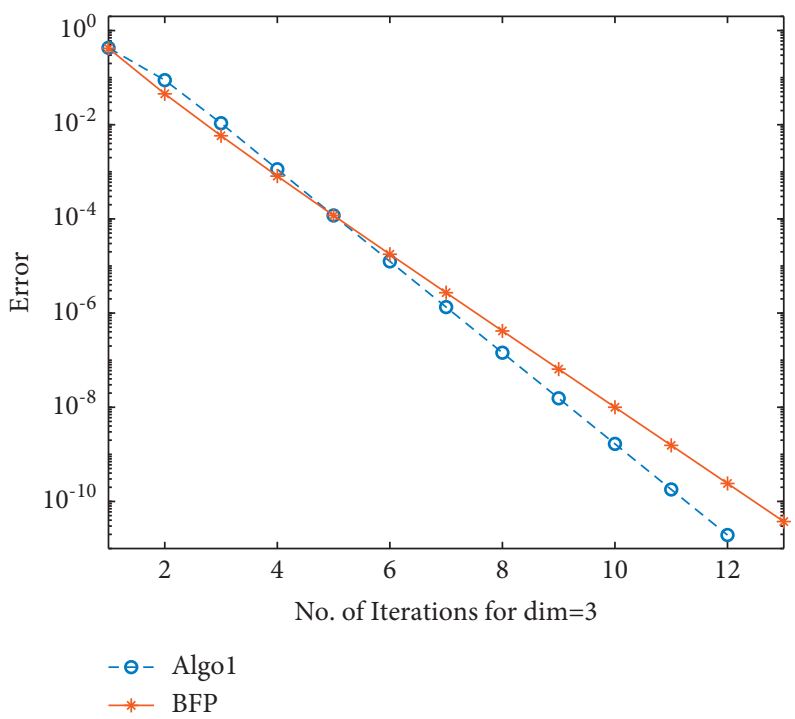

FIgURe 9: Iteration no. vs error for dimension 5. 

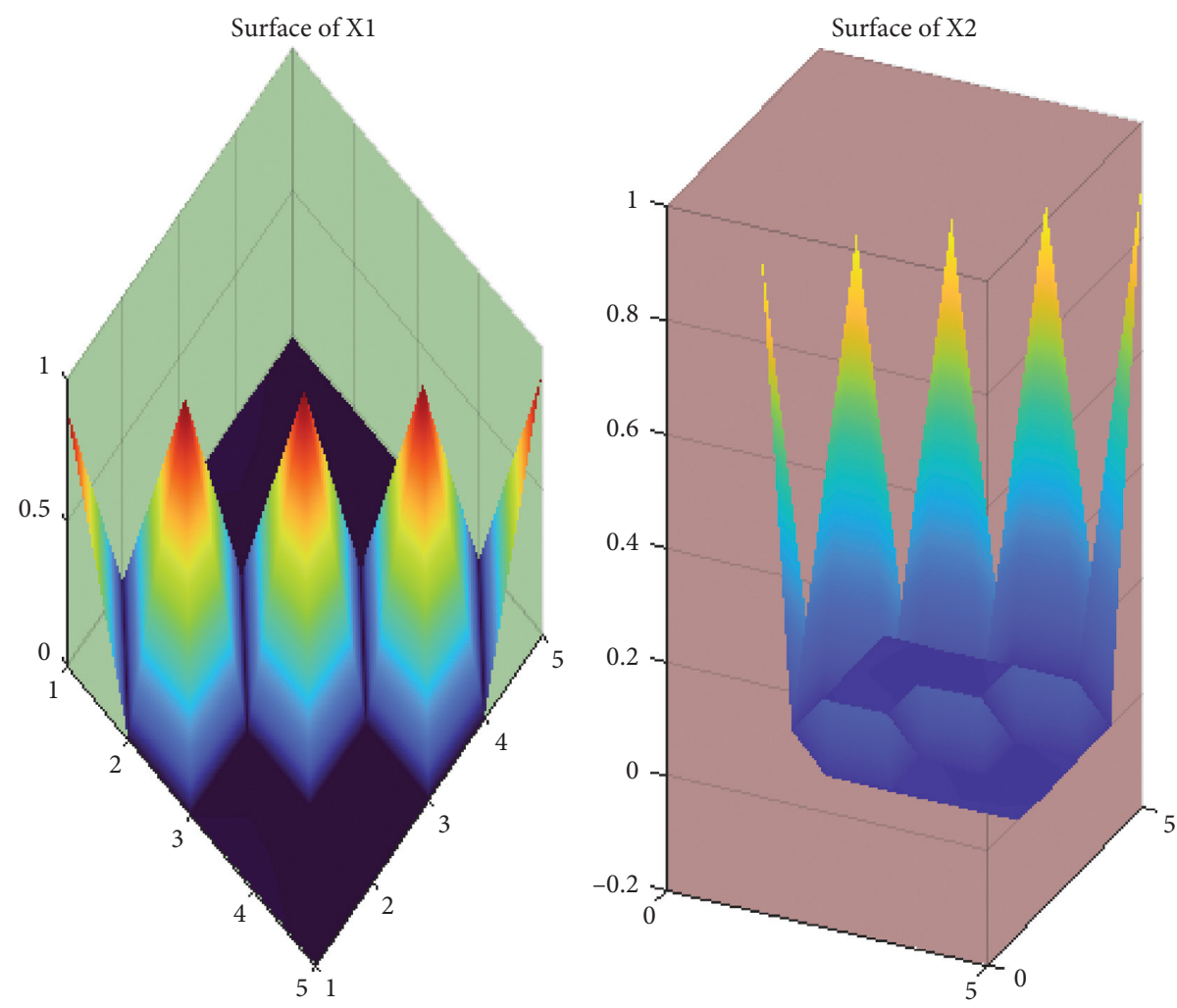

FIGURE 10: Solution surface plot for dimension 5.

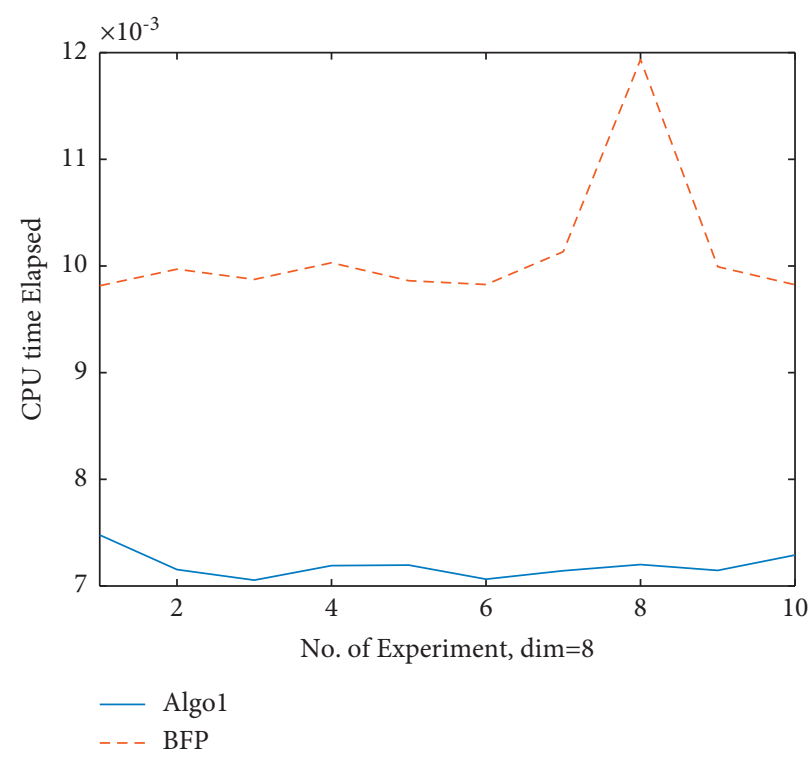

Figure 11: CPU time vs no. of iteration for dimension 8 . 


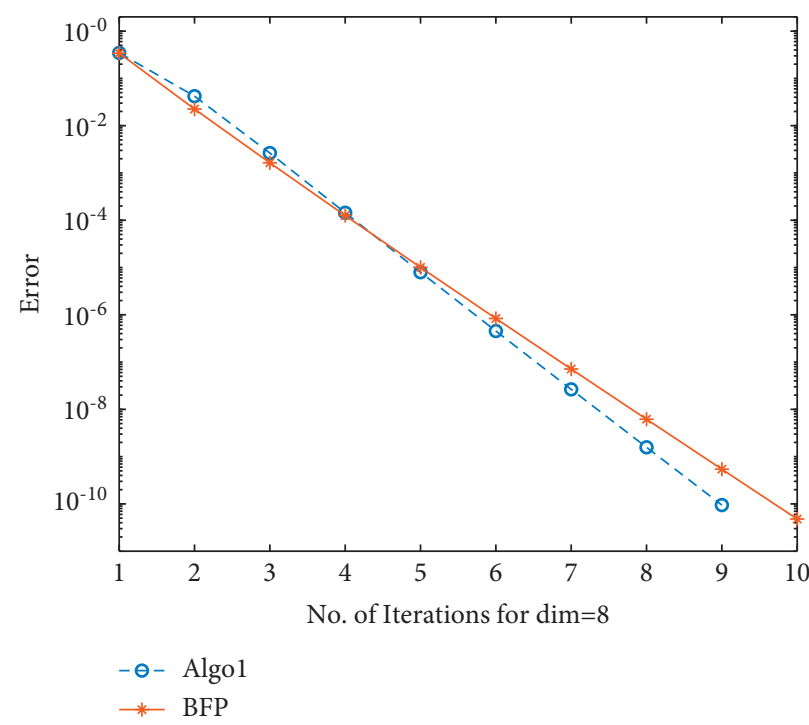

FIGURE 12: Iteration no. vs error for dimension 8.
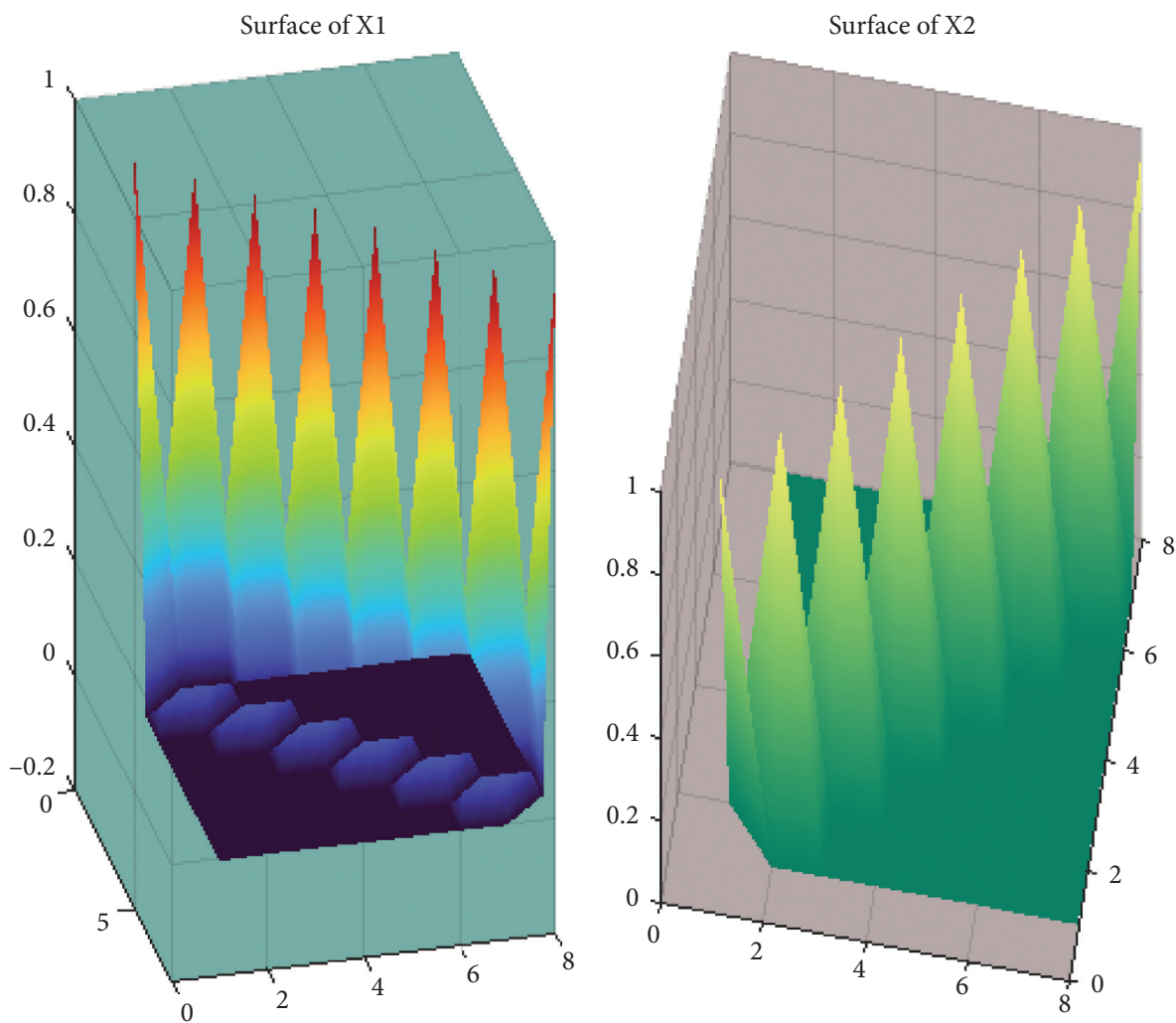

Figure 13: Solution surface plot for dimension 8 . 


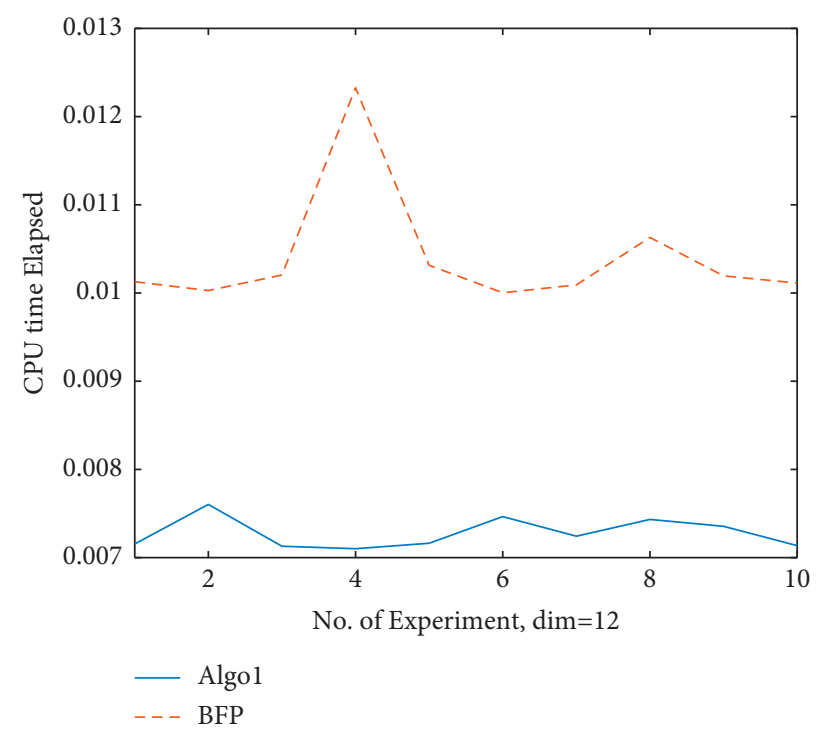

Figure 14: CPU time vs no of iteration for dimension 12.

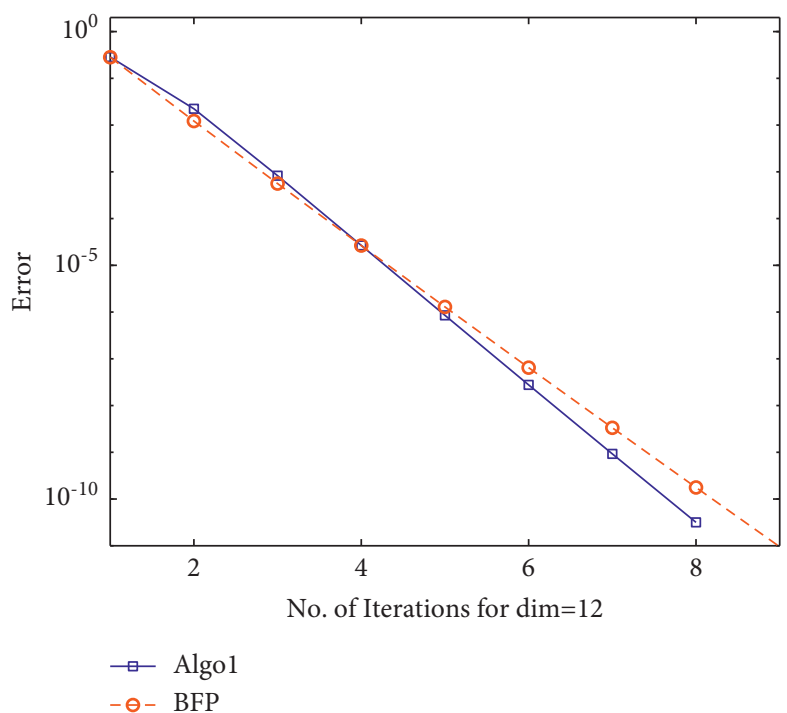

Figure 15: Iteration no. vs error for dimension 12. 

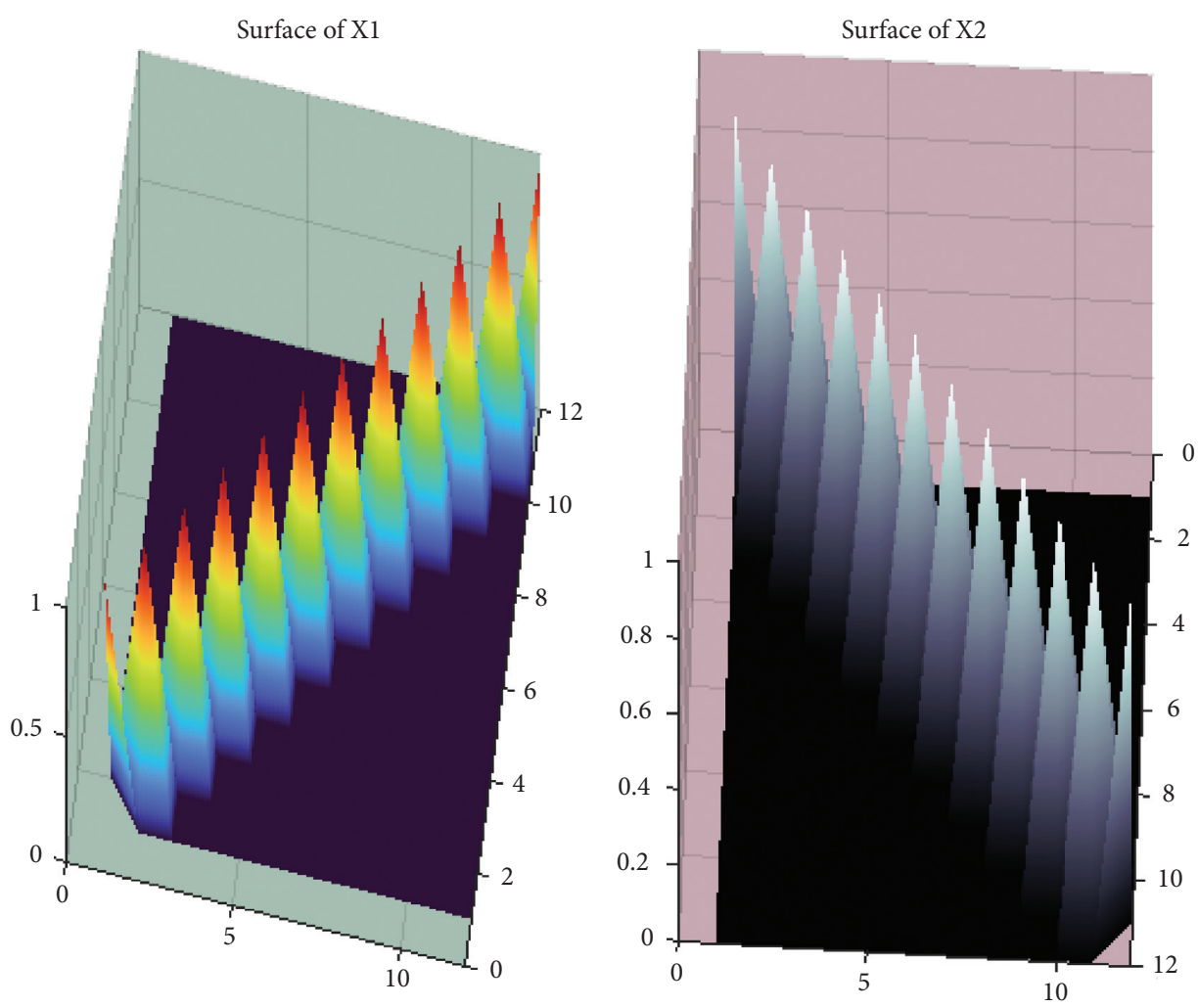

FIGURE 16: Solution surface plot for dimension 12.

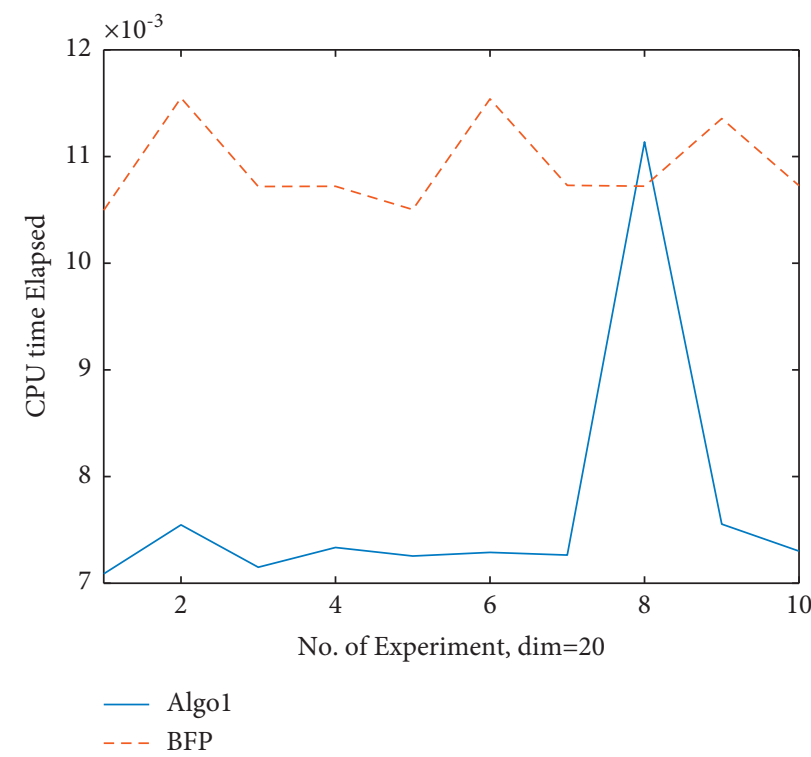

FIGURE 17: CPU time vs no. of iteration for dimension 20. 


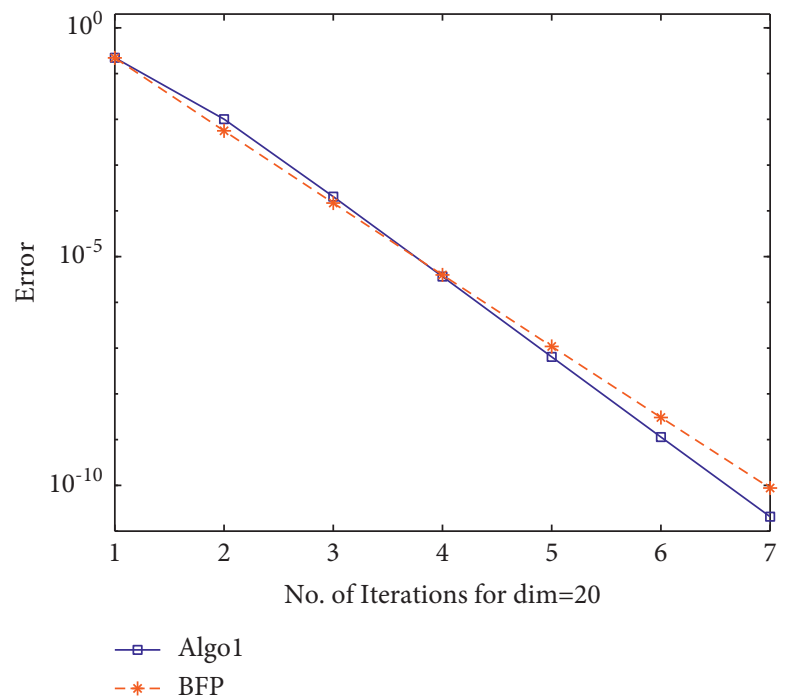

FIGURE 18: Iteration no. vs error for dimension 20.
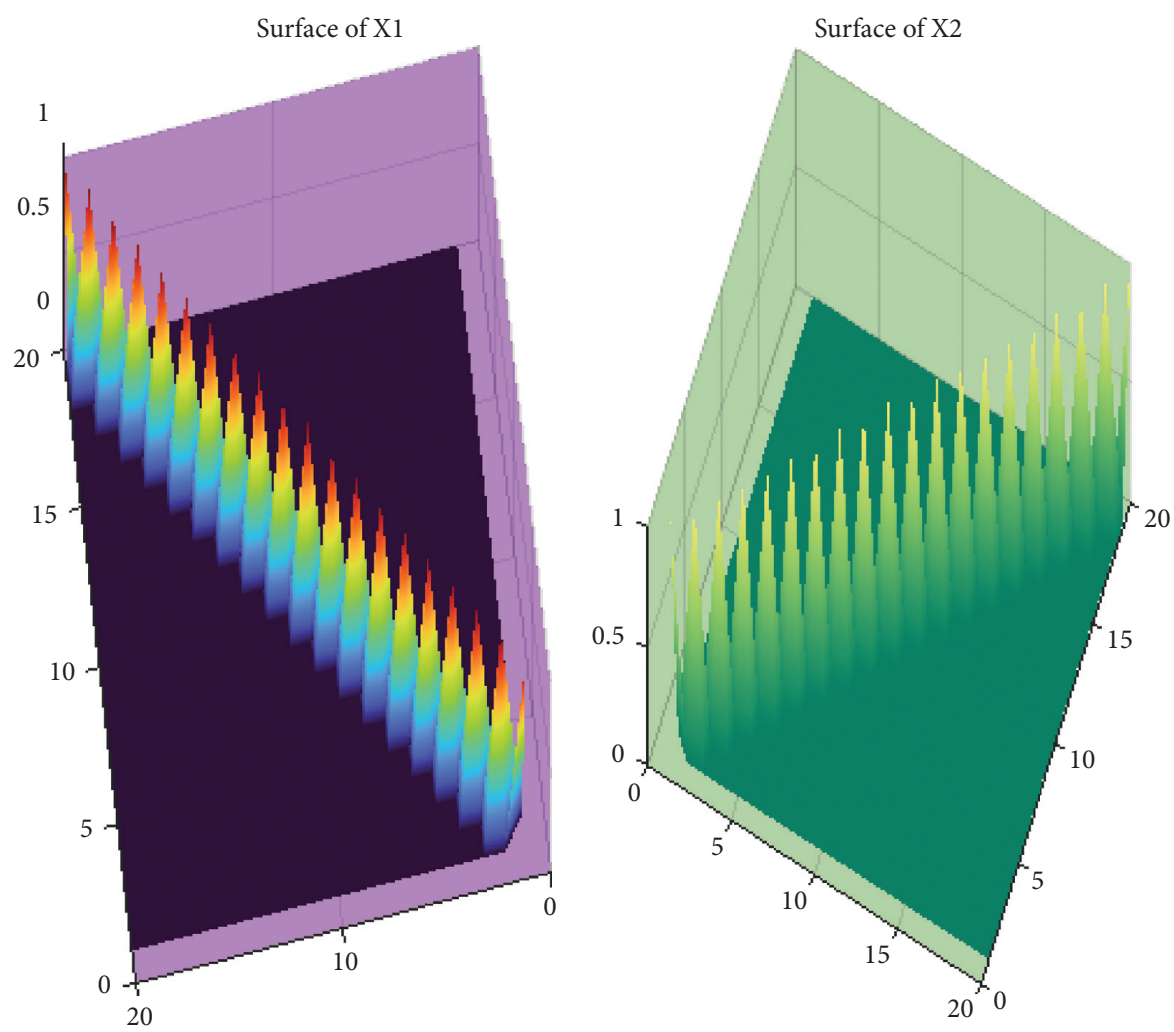

Figure 19: Solution surface plot for dimension 20. 


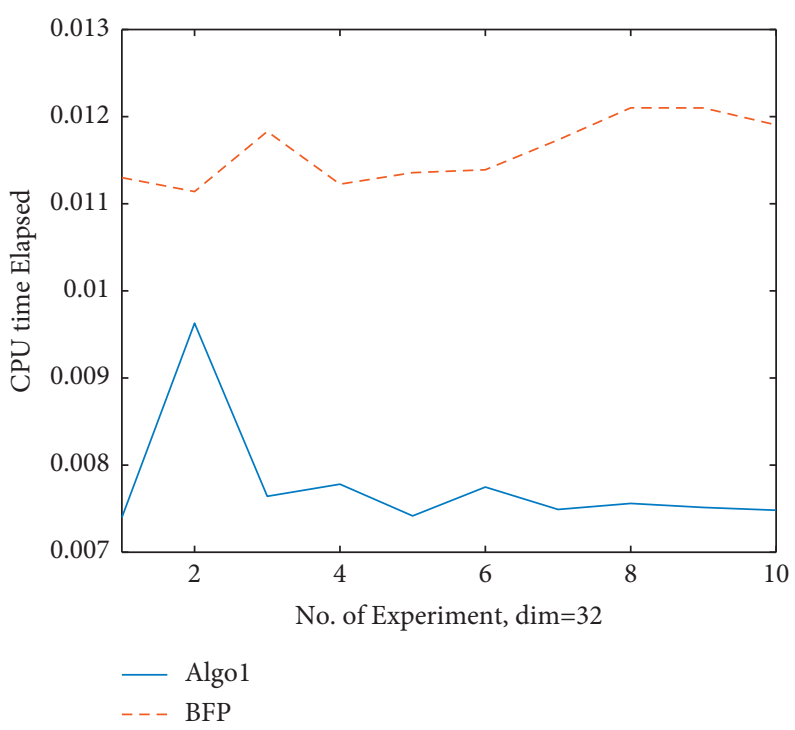

FIgURe 20: CPU time vs no. of iteration for dimension 32.

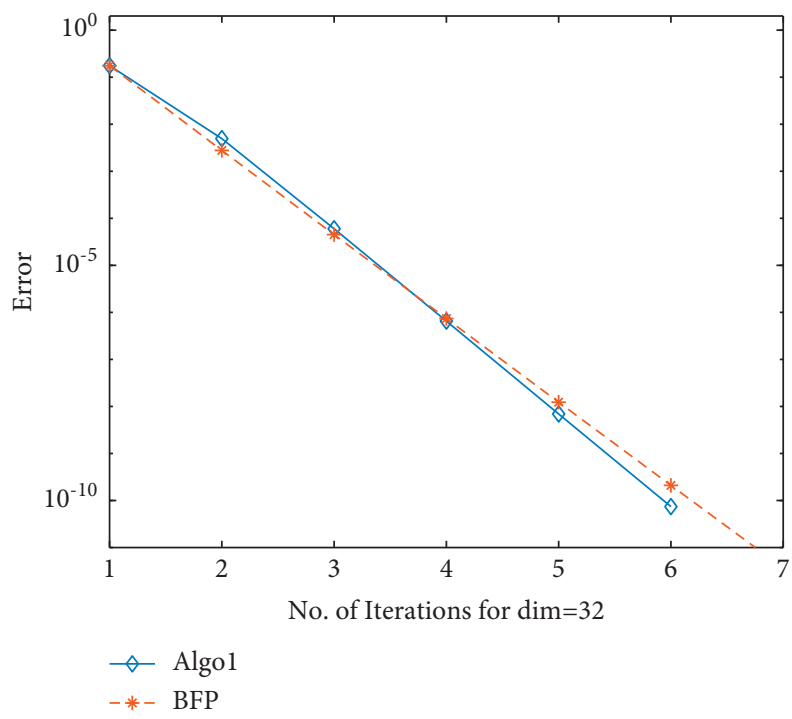

Figure 21: Iteration no. vs error for dimension 32. 

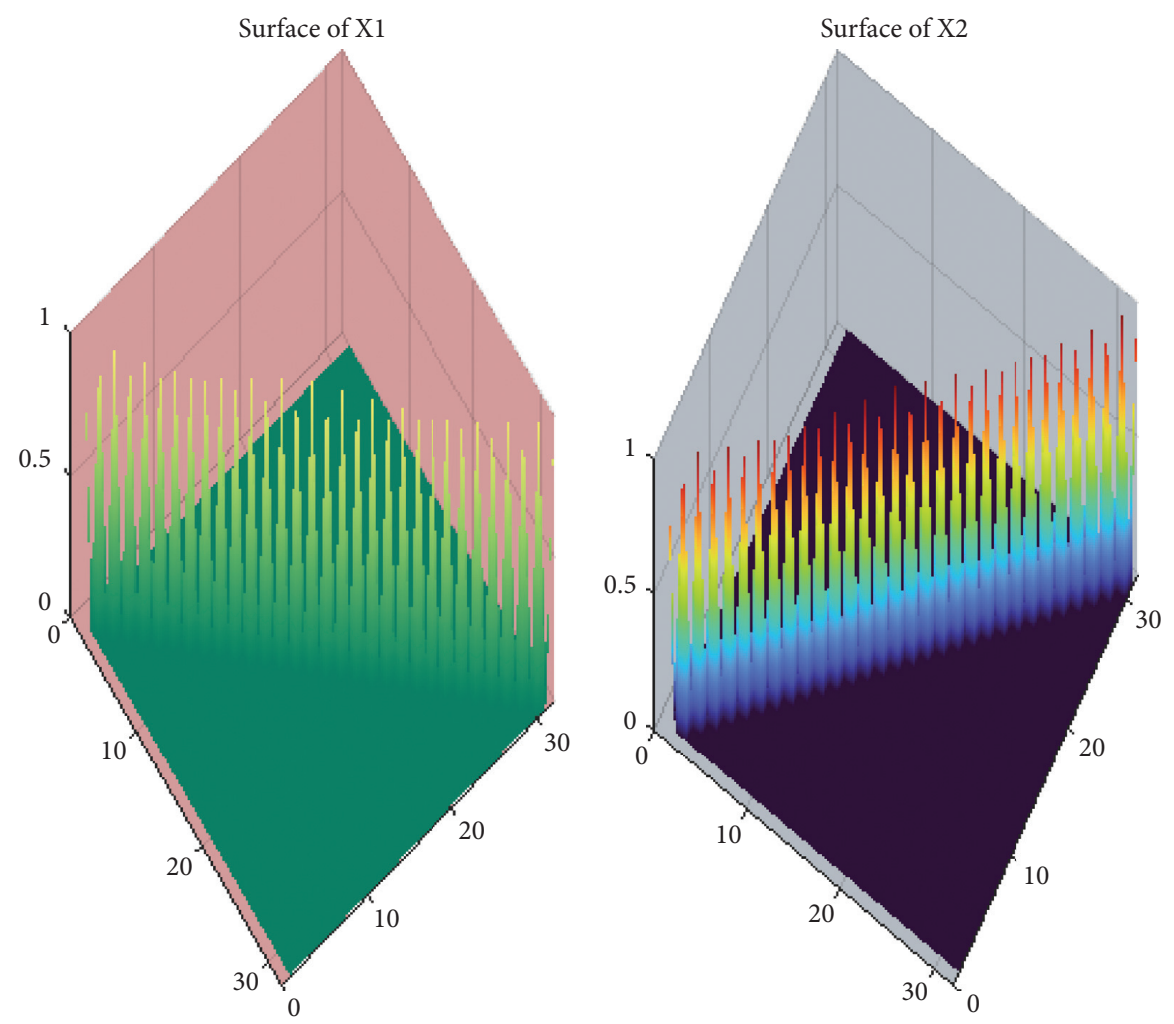

Figure 22: Solution surface plot for dimension 32.

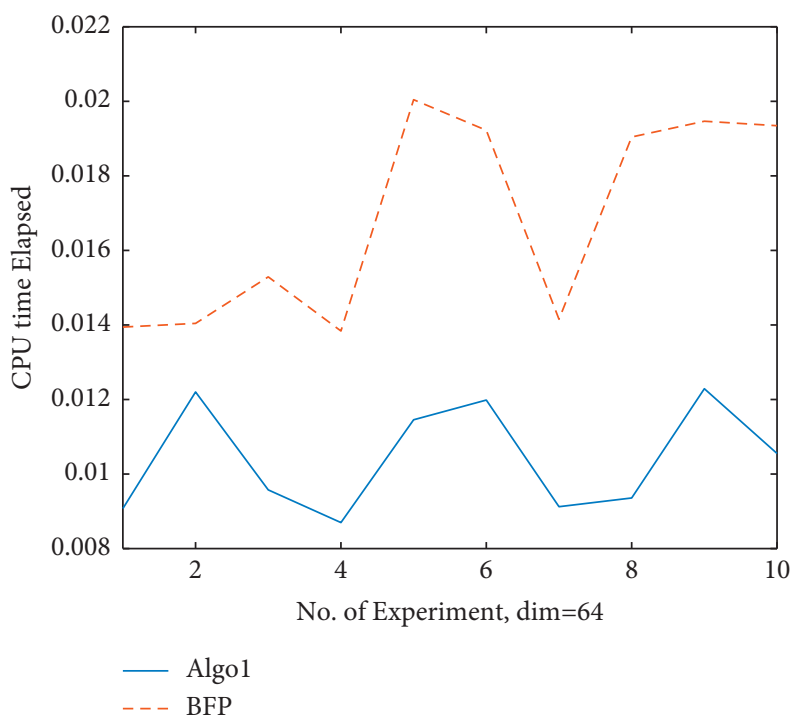

Figure 23: CPU time vs no. of iteration for dimension 64 . 


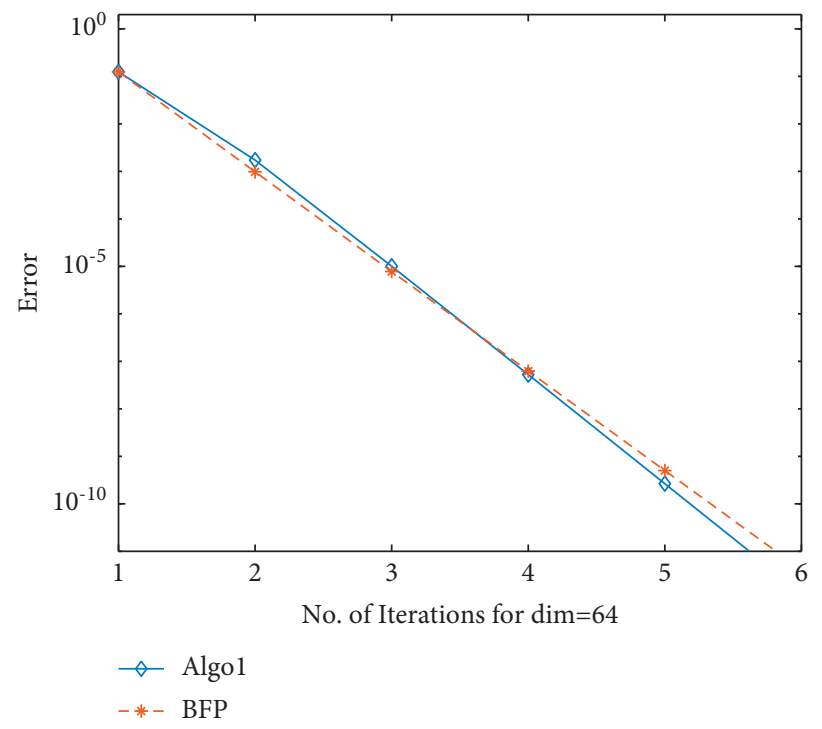

Figure 24: Iteration no. vs error for dimension 64 .
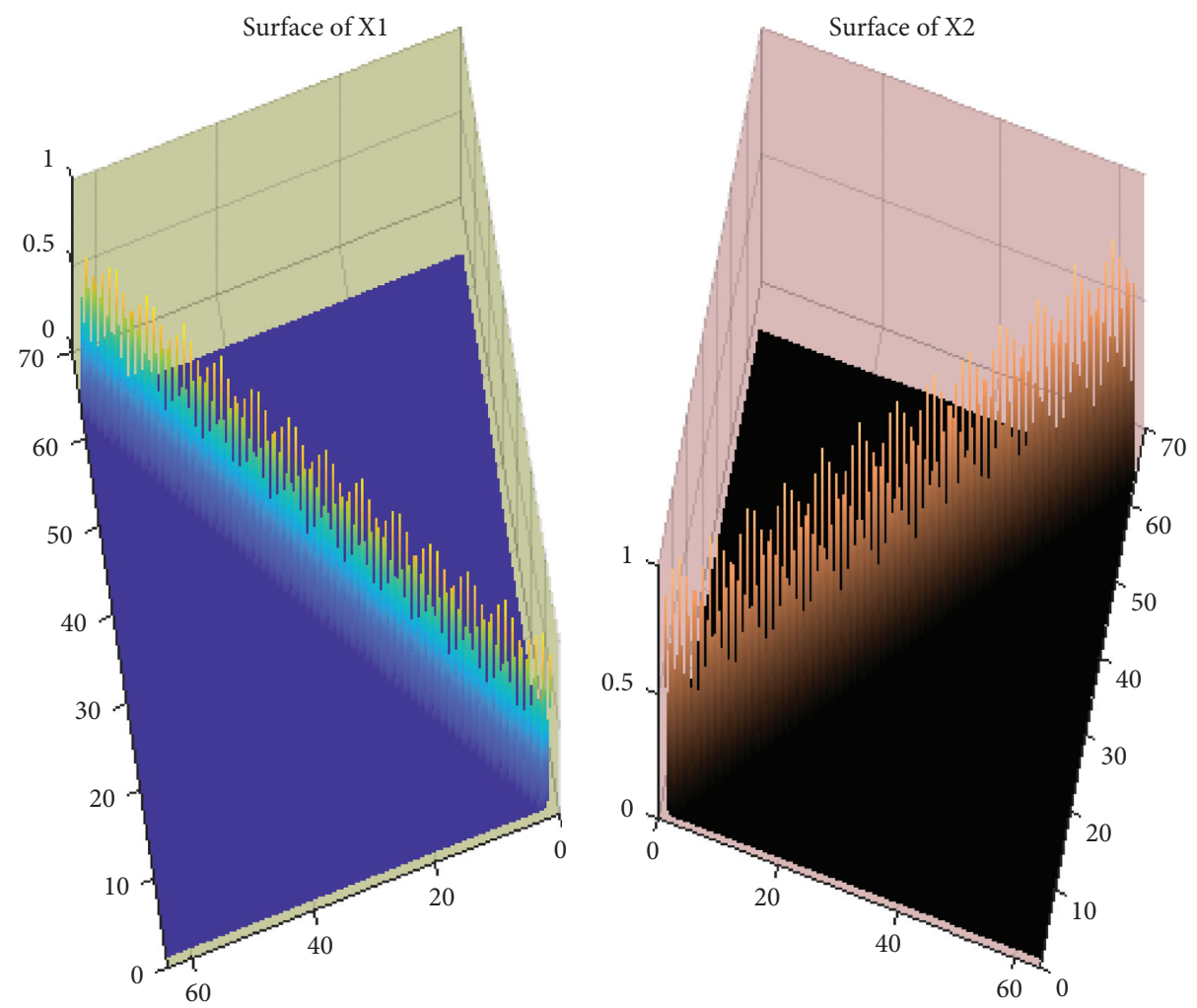

FiguRE 25: Solution surface plot for dimension 64 . 


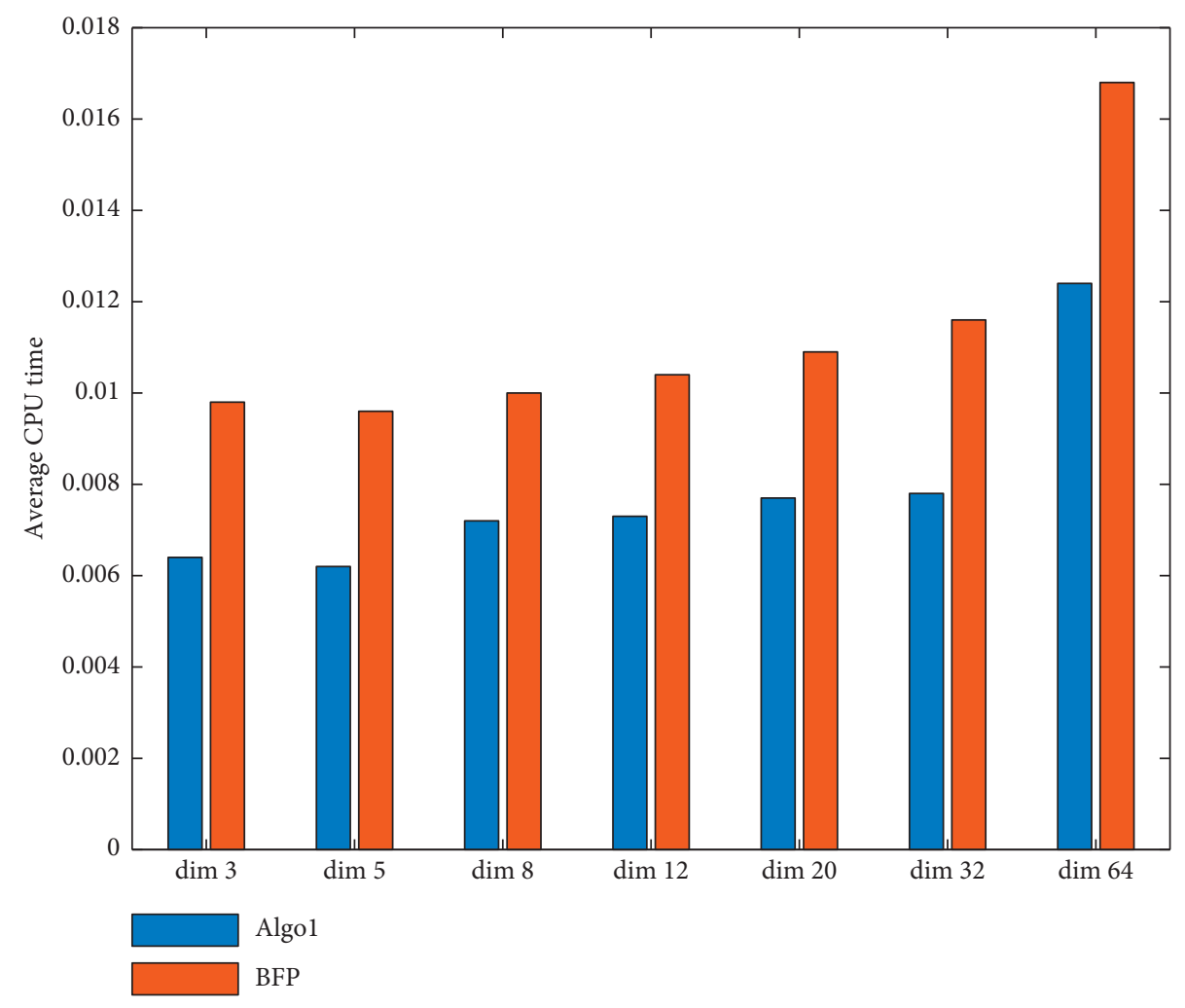

FIgure 26: Average CPU time for different dimensions.

\section{Conclusion}

In this study, a new iterative algorithm has been developed. All numerical tests are in agreement with the theoretical findings of this research done. Finally, based on the numerical results, we have concluded that the new iterative approach is extremely powerful and efficient in finding numerical solutions for a wide range of nonlinear matrix equations including complex ones. It also produces very accurate results with less iterations and lower computational costs, compared with the basic fixed-point approach.

\section{Data Availability}

No data were used to support the findings of the study.

\section{Conflicts of Interest}

The authors declare that they have no conflicts of interest.

\section{Acknowledgments}

The authors are grateful to the Science and Engineering Research Board (SERB), India, for providing project funding under the scheme-CRG/2018/000615.

\section{References}

[1] A. Liu and G. Chen, "On the hermitian positive definite solutions of nonlinear matrix equation $X^{s}+A^{*} X^{-t_{1}} A+B^{*} X^{-t_{2}} B=Q$," Mathematical Problems in Engineering, vol. 3, p. 18, Article ID 163585, 2011.
[2] N. Huang and C. M. Ma, "The inversion-free iterative methods for solving the nonlinear matrix equation $X=I-A^{H} X^{-1} A-B^{H} X^{-1} B$, , Abstract and Applied Analysis, vol. 2013, Article ID 843785, 7 pages, 2013.

[3] J.-h. Long, X.-y. Hu, and L. Zhang, "On the Hermitian positive defnite solution of the nonlinear matrix equation $\mathrm{X}+\mathrm{A}^{*} \mathrm{X}^{-1} \mathrm{~A}$ $+\mathrm{B}^{*} \mathrm{X}^{-1} \mathrm{~B}=\mathrm{I}$, , Bulletin of the Brazilian Mathematical Society, New Series, vol. 39, no. 3, pp. 371-386, 2008.

[4] R. Erfanifar, K. Sayevand, and H. Esmaeili, "A novel iterative method for the solution of a nonlinear matrix equation," Applied Numerical Mathematics, vol. 153, pp. 503-518, 2020.

[5] V. I. Hasanov and A. A. Ali, "On convergence of three iterative methods for solving of the matrix equation $\mathrm{X}+\mathrm{A}^{*} \mathrm{X}^{-1} \mathrm{~A}+\mathrm{B}^{*} \mathrm{X}^{-1} \mathrm{~B}=\mathrm{Q}$," Computational and Applied Mathematics, vol. 36, no. 1, pp. 79-87, 2017.

[6] S. Vaezzadeh, S. M. Vaezpour, R. Saadati, and C. Park, "The iterative methods for solving nonlinear matrix equation, $X+A^{H} X^{-1} A+B^{H} X^{-1} B=Q$," Advances in Difference Equations, vol. 2013, Article ID 229, 2013.

[7] S. Pakhira, S. Bose, and S. M. Hossein, "Solution of a class of nonlinear matrix equations," Bulletin of the Iranian Mathematical Society, vol. 47, no. 2, pp. 415-434, 2021.

[8] H. Ali and S. M. Hossein, "On the positive definite solution of a class of pair of nonlinear matrix equations," Computational and Applied Mathematics, vol. 39, no. 102, 2020.

[9] A. M. Al-Dubiban, "Iterative algorithm for solving a system of nonlinear matrix equations," Journal of Applied Mathematics, vol. 2012, Article ID 461407, 15 pages, 2012.

[10] A. M. Al-Dubiban, "On the iterative method for the system of nonlinear matrix equations," Abstract and Applied Analysis, vol. 2013, Article ID 685753, 7 pages, 2013.

[11] N. Huang, C. Ma, and J. Tang, "The inversion-free iterative methods for a system of nonlinear matrix equations," 
International Journal of Computer Mathematics, vol. 93, no. 9, pp. 1470-1483, 2016.

[12] Z. Asgari, F. Toutounian, E. Babolian, and E. Tohidi, “An extended block Golub-Kahan algorithm for large algebraic and differential matrix Riccati equations," Computers \& Mathematics with Applications, vol. 79, no. 8, pp. 2447-2457, 2020.

[13] E. Tohidi and M. Khorsand Zak, "A new matrix approach for solving second-order linear matrix partial differential equations," Mediterranean Journal of Mathematics, vol. 13, no. 3, pp. 1353-1376, 2016.

[14] Z. Y. Peng, S. M. El-sayed, and X. L. Zhang, "Iterative methods for the extremal positive definite solution of the matrix equation $X+A^{*} X^{-\alpha} A=Q$," Journal of Computational and Applied Mathematics, vol. 200, pp. 520-527, 2007.

[15] D. M. Zhou, G. I. Chen, G. X. Wu, and X. Y. Zhang, "Some properties of the nonlinear matrix equation, $X^{s}+A^{*} X^{-t} A=Q$," Journal of Mathematical Analysis and Applications, vol. 392, pp. 75-82, 2012.

[16] T. Furuta, "Operator inequalities associated with Holder-McCarthy and Kantorovich inequalities," Journal of Inequalities and Applications, vol. 6, pp. 137-148, 1998.

[17] R. A. Horn and C. R. Johnson, Matrix Analysis, Cambridge University Press, Cambridge, UK, second edition, 2012.

[18] R. Bhatia, Positive Definite Matrices, Princeton University Press, Princeton, NJ, USA, 2007. 